ARTICLE

\title{
Fluid flow-induced left-right asymmetric decay of Dand5 mRNA in the mouse embryo requires a Bicc1-Ccr4 RNA degradation complex
}

Katsura Minegishi (iD) 1,7, Benjamin Rothé2,7, Kaoru R. Komatsu (iD 3,7, Hiroki Ono ${ }^{3}$, Yayoi Ikawa1, Hiromi Nishimura (10 1, Takanobu A. Katoh (10 1, Eriko Kajikawa1, Xiaorei Sai ${ }^{1}$, Emi Miyashita ${ }^{3}$, Katsuyoshi Takaoka', Kana Bando ${ }^{4}$, Hiroshi Kiyonari (i] ${ }^{4}$, Tadashi Yamamoto (1) ${ }^{5,6}$, Hirohide Saito (1) ${ }^{3 凶}$, Daniel B. Constam (1) ${ }^{2 凶} \&$ Hiroshi Hamada (1D) ${ }^{1 凶}$

Molecular left-right (L-R) asymmetry is established at the node of the mouse embryo as a result of the sensing of a leftward fluid flow by immotile cilia of perinodal crown cells and the consequent degradation of Dand5 mRNA on the left side. We here examined how the fluid flow induces Dand5 mRNA decay. We found that the first 200 nucleotides in the $3^{\prime}$ untranslated region ( $3^{\prime}-$ UTR) of Dand5 mRNA are necessary and sufficient for the left-sided decay and to mediate the response of a $3^{\prime}-$ UTR reporter transgene to $\mathrm{Ca}^{2+}$, the cation channel Pkd2, the RNA-binding protein Biccl and their regulation by the flow direction. We show that Biccl preferentially recognizes GACR and YGAC sequences, which can explain the specific binding to a conserved GACGUGAC motif located in the proximal Dand5 3'-UTR. The Cnot3 component of the Ccr4-Not deadenylase complex interacts with Biccl and is also required for Dand5 mRNA decay at the node. These results suggest that $\mathrm{Ca}^{2+}$ currents induced by leftward fluid flow stimulate Bicc1 and Ccr4-Not to mediate Dand5 mRNA degradation specifically on the left side of the node.

\footnotetext{
${ }^{1}$ Laboratory for Organismal Patterning, RIKEN Center for Biosystems Dynamics Research, Kobe, Hyogo, Japan. ${ }^{2}$ Ecole Polytechnique Fédérale de Lausanne (EPFL), School of Life Sciences, Lausanne, Switzerland. ${ }^{3}$ Department of Life Science Frontiers, Center for iPS Cell Research and Application (CiRA), Kyoto University, Kyoto, Japan. ${ }^{4}$ Laboratory for Animal Resources and Genetic Engineering, RIKEN Center for Biosystems Dynamics Research, Kobe, Hyogo, Japan. ${ }^{5}$ Laboratory for Immunogenetics, Center for Integrative Medical Sciences, Suehiro-cho, Yokohama, Japan. ${ }^{6}$ Cell Signal Unit, Okinawa Institute of Science and Technology, Kunigami-gun, Okinawa, Japan. ${ }^{7}$ These authors contributed equally: Katsura Minegishi, Benjamin Rothé, Kaoru R. Komatsu. ${ }^{凶}$ email: hirohide. saito@cira.kyoto-u.ac.jp; daniel.constam@epfl.ch; hiroshi.hamada@riken.jp
} 
T he first step in the establishment of left-right (L-R) asymmetry in vertebrate embryos is known as the L-R symmetry breaking event. In fish, frog, and mouse embryos, the breaking of L-R symmetry requires motile cilia that generate a unidirectional fluid flow at the L-R organizer, which corresponds to Kupffer's vesicle in zebrafish, the gastrocoel roof plate in Xenopus, and the ventral node in mouse ${ }^{1,2}$. Whereas this fluid flow, known as nodal flow in the mouse, is essential for L-R symmetry breaking, its mechanism of action remains unknown. It may transport an unknown chemical determinant or it may generate a mechanical stimulus, either of which would then be sensed by the embryo.

Genetic studies have indicated that nodal flow is sensed by the transient receptor potential (TRP) cation channel $\mathrm{Pkd} 2^{3}$ and its partner protein $\mathrm{Pkd} 111^{4}$ present in immotile cilia of crown cells at the periphery of the node ${ }^{5}$. Flow sensation by $\mathrm{Pkd} 2$ is thought to result in the entry of $\mathrm{Ca}^{2+6}$ and the consequent degradation through an unknown mechanism of the mRNA for Dand5 (also known as Cerl2 or Cer2) in crown cells on the left side of the node ${ }^{1,7}$. Given that Dand5 is an antagonist of the extracellular signaling molecule Nodal, which is required for correct L-R patterning 8 , suppression of Dand5 mRNA on the left side results in increased Nodal activity in crown cells on this side 9 . Flowinduced downregulation of Dand5 mRNA specifically on the left side is the earliest molecular L-R asymmetry that has been detected at the node, and it, in turn, induces asymmetric expression of Nodal in the lateral plate ${ }^{9}$.

The specific factors responsible for the suppression of Dand5 mRNA have remained unidentified. The only RNA binding protein known to be required for correct $\mathrm{L}-\mathrm{R}$ patterning is Bicc $1^{10}$. Biccl binds specific mRNAs via a tandem repeat of three ribonucleoprotein $\mathrm{K}$ homology $(\mathrm{KH})$ domains and thereby inhibits their translation in a manner that is facilitated by selfpolymerization of a sterile a motif (SAM) at the $\mathrm{COOH}$-terminus of the protein ${ }^{11,12}$. Studies in Drosophila have shown that Bicc1 also interacts with the Ccr4-Not complex-the major cytoplasmic deadenylase responsible for mRNA turnover in eukaryotes ${ }^{13,14}$ through direct binding to its Not3/5 subunit ${ }^{15}$. In mammalian cells, lack of the Not3/5 ortholog Cnot3 is associated with increased mRNA stability ${ }^{16,17}$. However, a function for Biccl or the Ccr4-Not deadenylase complex in flow sensing, or in the regulation of mRNA translation or decay, during L-R patterning has not been described.

We have now identified an element in the $3^{\prime}$ untranslated region ( $3^{\prime}$-UTR) of Dand5 mRNA that is responsible for the asymmetric decay of this mRNA at the node of the mouse embryo in a manner dependent on Biccl and the Ccr4-Not subunit Cnot3.

\section{Results}

A $3^{\prime}$-UTR reporter recapitulates the asymmetric distribution of Dand5 mRNA at the node. The L-R pattern of Dand5 mRNA changes dynamically at the node of mouse embryos. At the early headfold stage, Dand5 mRNA is induced specifically in crown cells on both sides of the node, and its distribution remains symmetric until the zero-somite stage (see Fig. 1). At the threesomite stage, the amount of Dand5 mRNA has begun to decrease on the left side, resulting in a L-R asymmetric $(\mathrm{R}>\mathrm{L})$ distribution that is maintained at least until the six-somite stage. This decline in the abundance of Dand5 mRNA on the left side coincides with a gradual increase in nodal flow ${ }^{9,18}$. Such observations have thus suggested that Dand5 mRNA is degraded in crown cells on the left side of the node between the zero- and five-somite stages.

We first tested if the $3^{\prime}$-UTR of Dand5 mRNA is able to recapitulate the distribution pattern of the mRNA at the node with the use of a transgene, NDE-Hsp-dsVenus-3'-UTR, that contains the open reading frame (ORF) for destabilized Venus (dsVenus) linked to the 1.2-kb DNA sequence corresponding to the $3^{\prime}$-UTR of Dand5 mRNA (Fig. 1a). Expression of the $d s$ Venus- $3^{\prime}-U T R$ cassette is driven by the mouse Hsp68 promoter and the crown cell-specific enhancer (NDE) of mouse Nodal. As expected, NDE-Hsp-dsVenus-3'-UTR transgenic embryos expressed dsVenus specifically in node crown cells. Both $d s V e n u s$ mRNA (Fig. 1c) and dsVenus fluorescence (Fig. 1a and d) were bilaterally equal at the early headfold and zero-somite stages but were asymmetric $(\mathrm{R}>\mathrm{L})$ at the three- and five-somite stages, recapitulating the pattern of Dand5 mRNA (Fig. $1 \mathrm{~b}$ and e). Hereafter, asymmetry of dsVenus fluorescence was examined at the five- to six-somite stages when the L-R asymmetry becomes obvious (Fig. 1e) unless indicated otherwise.

The $3^{\prime}$-UTR of Dand5 mRNA responds to the direction of fluid flow in a Pk d2-dependent manner. To test whether the NDEHsp-dsVenus- $3^{\prime}-U T R$ transgene is regulated by nodal flow, we introduced it into iv/iv (Dnah1 $11^{i v / i v}$ ) mutant embryos, which lack the fluid flow ${ }^{19}$. In such embryos $(n=6 / 6)$, the transgene failed to give rise to an asymmetric $(\mathrm{R}>\mathrm{L})$ distribution of dsVenus fluorescence (Fig. 2a). Interestingly, the dsVenus pattern is bilaterally equal in all embryos examined (Fig. 2b and b), although L-R is often described as being randomized. This discrepancy may be due to the sensitivity of the assay employed. Mouse embryos in culture develop L-R asymmetry in response to the direction of an artificial fluid flow ${ }^{20}$. Exposure of wild-type (WT) embryos harboring the transgene to an artificial leftward fluid flow resulted in the development of $\mathrm{R}>\mathrm{L}$ asymmetry of dsVenus fluorescence $(n=6 / 8)$ (Fig. $2 c$ and d). By contrast, when exposed to an artificial flow toward the right side, such embryos instead developed bilaterally symmetric $(\mathrm{R}=\mathrm{L})$ dsVenus fluorescence $(n=6 / 6)$. Exposure of $i v /+$ or $i v / i v$ embryos to the artificial rightward flow resulted in the development of $\mathrm{R}<\mathrm{L}$ $(n=3 / 6), \mathrm{R}=\mathrm{L}(n=2 / 6)$, or $\mathrm{R}>\mathrm{L}(n=1 / 6)$ patterns of dsVenus fluorescence in the former embryos and in $\mathrm{R}<\mathrm{L}(n=2 / 7), \mathrm{R}=\mathrm{L}$ $(n=4 / 7)$, and $\mathrm{R}>\mathrm{L}(n=1 / 7)$ patterns in the latter (Fig. $2 \mathrm{c}$ and $\mathrm{d}$, Supplementary Fig. 1a). These results collectively suggested that the $3^{\prime}$-UTR of Dand5 mRNA responds to the direction of fluid flow.

$P k d 2$ encodes an ion channel that allows the passage of cations including $\mathrm{Ca}^{2+21}$, and is required for $\mathrm{L}-\mathrm{R}$ patterning in the mouse embryo ${ }^{3}$. In $P k d 2^{-/-}$embryos, the NDE-Hsp-dsVenus- $3^{\prime}$ UTR transgene was expressed bilaterally at the node $(n=7 / 7)$ (Fig. 2e, f). Similarly, treatment of WT embryos harboring the transgene for $1 \mathrm{~h}$ with thapsigargin, which blocks $\mathrm{Ca}^{2+}$ release from the endoplasmic reticulum ${ }^{22}$, resulted in half of the tested embryos $(n=7 / 14)$ failing to downregulate the reporter mRNA on the left side in response to an artificial leftward flow, disrupting the L-R asymmetric pattern of dsVenus fluorescence (Fig. $2 \mathrm{~g}$ and $\mathrm{h}$ ). Longer treatment with thapsigargin resulted in developmental arrest. Together, these results implicate $\mathrm{Ca}^{2+}$ in the flow-induced decay of Dand5 mRNA.

A conserved 200-nucleotide region of the proximal $3^{\prime}$-UTR is required for L-R asymmetry of Dand5 mRNA. To map the sequence within the $3^{\prime}$-UTR required for generation of L-R asymmetry of Dand5 mRNA, we first compared the DNA sequences corresponding to the $3^{\prime}$-UTR $(\sim 1.2 \mathrm{~kb})$ among mammalian Dand5 genes. We found that the $5^{\prime}$-most 200 nucleotide region (200prox) was substantially conserved (Supplementary Fig. 2). To test whether this region is required for L-R asymmetric gene expression, we first deleted it from the NDE-Hsp-dsVenus$3^{\prime}$-UTR transgene (Fig. 3a). The resulting NDE-Hsp-dsVenus-3'- 
a
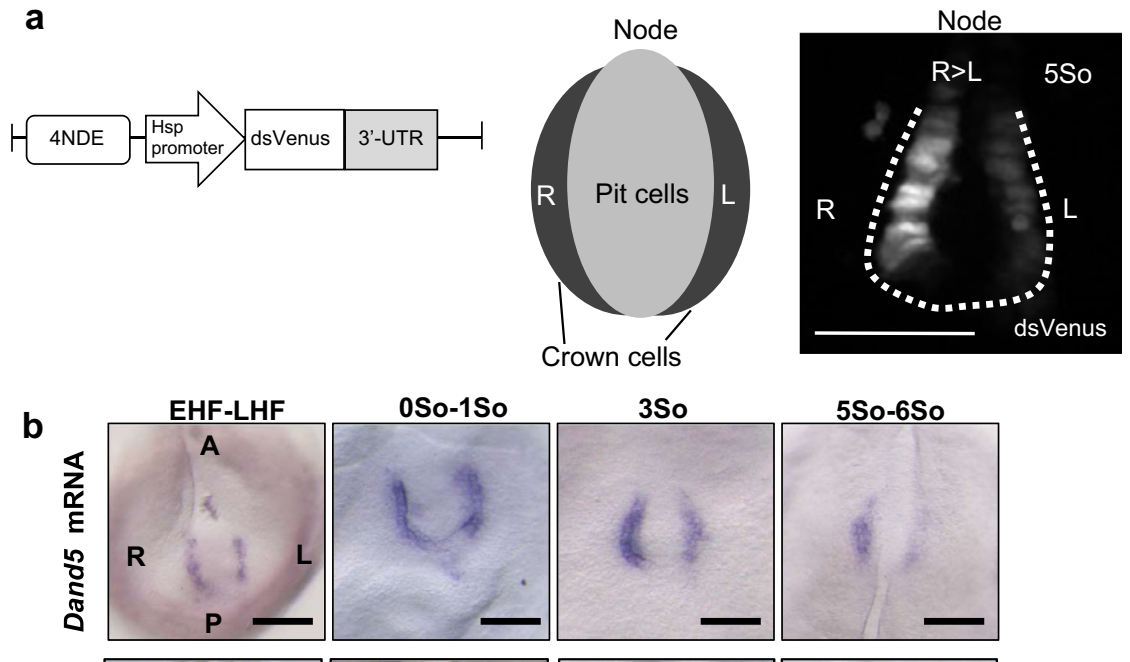

3So

5So-6So
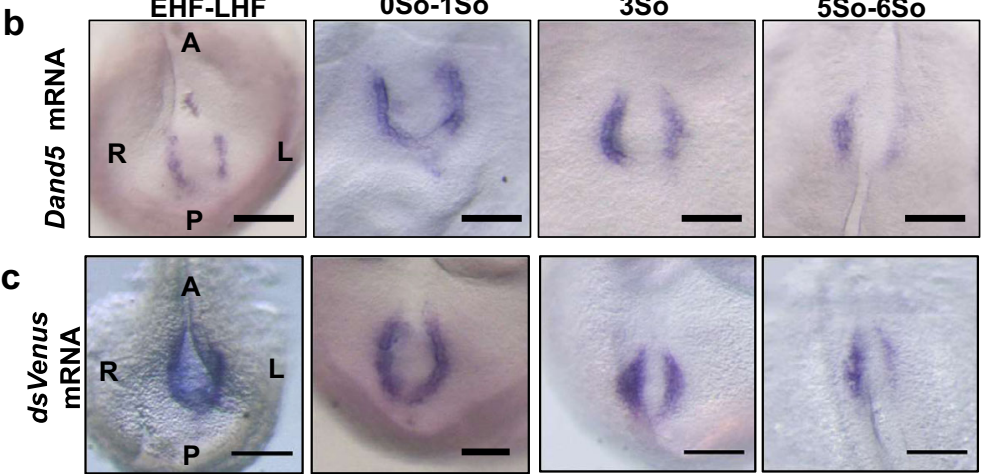

d
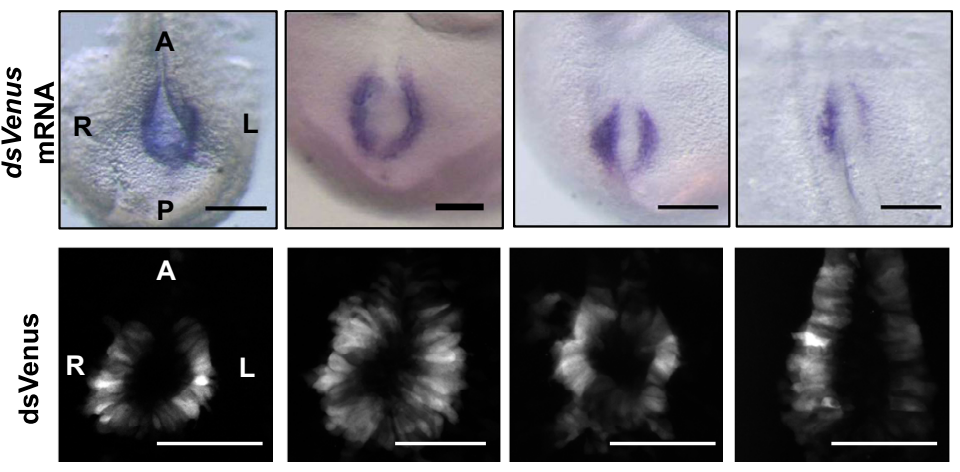

e

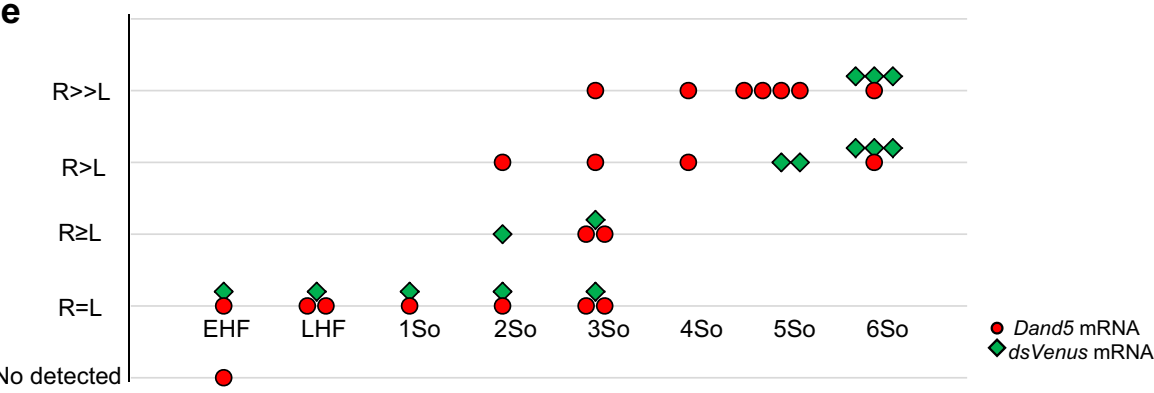

Fig. 1 A 3'-UTR reporter recapitulates the asymmetric distribution of Dand5 mRNA at the node. a Schematic representation of the NDE-Hsp-dsVenus-3'UTR transgene (left) and of the relative localizations of pit cells and crown cells at the node of mouse embryos (middle). The transgene contains the ORF for dsVenus linked to the DNA sequence for the 3'-UTR (1.2 kb) of mouse Dand5 mRNA, and its expression is driven by the mouse Hsp68 promoter and four copies of the crown cell-specific enhancer (NDE) of mouse Nodal. A typical pattern of dsVenus fluorescence detected at the node of a transgenic embryo at the five-somite (5So) stage is also shown (right), with the dashed line indicating the node region. Note that the fluorescence in crown cells is highly L-R asymmetric $(R>L)$. Scale bars, $100 \mu \mathrm{m}$. b Whole-mount in situ hybridization (WISH) analysis of Dand5 mRNA at the node of wild-type mouse embryos at the indicated developmental stages. Note that Dand5 mRNA in crown cells is bilaterally equal at the early headfold (EHF) to late headfold (LHF) stages as well as the zero- to one-somite stages, but is asymmetric $(R>L)$ at the three-somite and five- to six-somite stages. $A$ and $P$, anterior and posterior, respectively. Scale bars, $100 \mu \mathrm{m}$. c WISH analysis of dsVenus mRNA in NDE-Hsp-dsVenus-3'-UTR transgenic embryos at the indicated stages. Scale bars, $100 \mu \mathrm{m}$. d Fluorescence images of dsVenus at the node of transgenic embryos at the indicated stages. Scale bars, $100 \mu \mathrm{m}$. Images shown in (bd) are representative of at least two embryos at each stage. e Comparison of L-R asymmetry of Dand5 mRNA in wild-type embryos and dsVenus mRNA in transgenic embryos at various developmental stages. Each point corresponds to one embryo. Note that the pattern of $d s$ Venus mRNA recapitulates that of Dand5 mRNA.

UTR-A200prox transgene yielded bilaterally symmetric dsVenus fluorescence at the node (Fig. 3b, and Supplementary Fig. 1b) $(n$ $=5 / 6$ ). On the other hand, deletion of the $3^{\prime}$-most 200 nucleotides from the $3^{\prime}$-UTR ( $\Delta 200$ dist in Fig. 3a) did not perturb asymmetric dsVenus fluorescence (Fig. $3 b)(n=3 / 3)$. Furthermore, the $5^{\prime}$-most 200 nucleotide region (200prox in Fig. 3a) alone gave rise to asymmetric dsVenus fluorescence $(n=4 / 5)$ (Fig. 3b). Prompted by these results, we applied CRISPR-based editing to delete this proximal 200-nucleotide region of the Dand5 3 -UTR in the mouse genome, thereby generating the Dand5 ${ }^{\Delta 200 \text { prox }}$ allele (Supplementary Fig. 3). WISH analysis revealed that whereas bilateral Nodal expression at the node was maintained, left-sided Nodal expression in lateral plate mesoderm (LPM) was lost in Dand5 $5^{\Delta 200 p r o x / \Delta 200 p r o x}$ embryos $(n=4 / 4)$ (Fig. 3c), as expected if Dand5 mRNA remains stable on both sides of the node. Among eight Dand $5^{\Delta 200 \text { prox } /+}$ embryos 
a

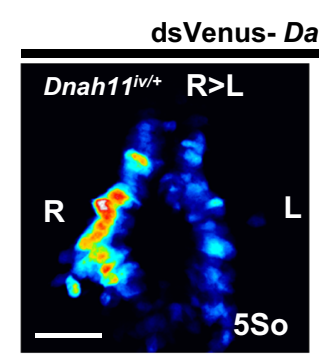

d

Artificial

leftward flow

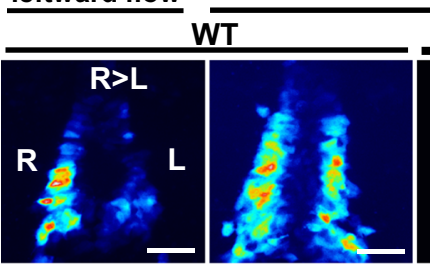

e

dsVenus- Dand5 3'-UTR
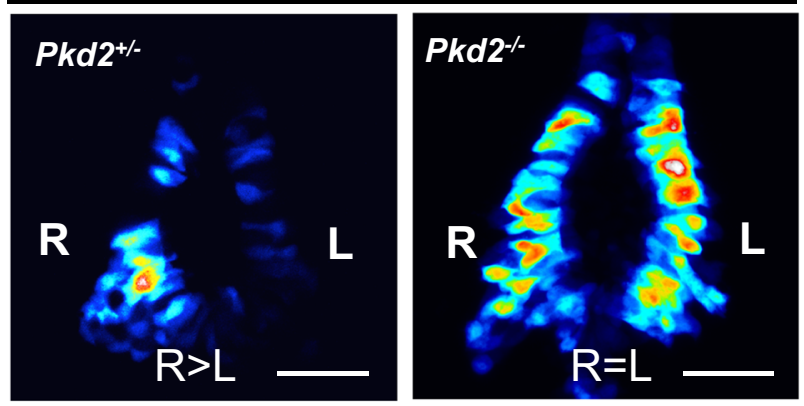

g
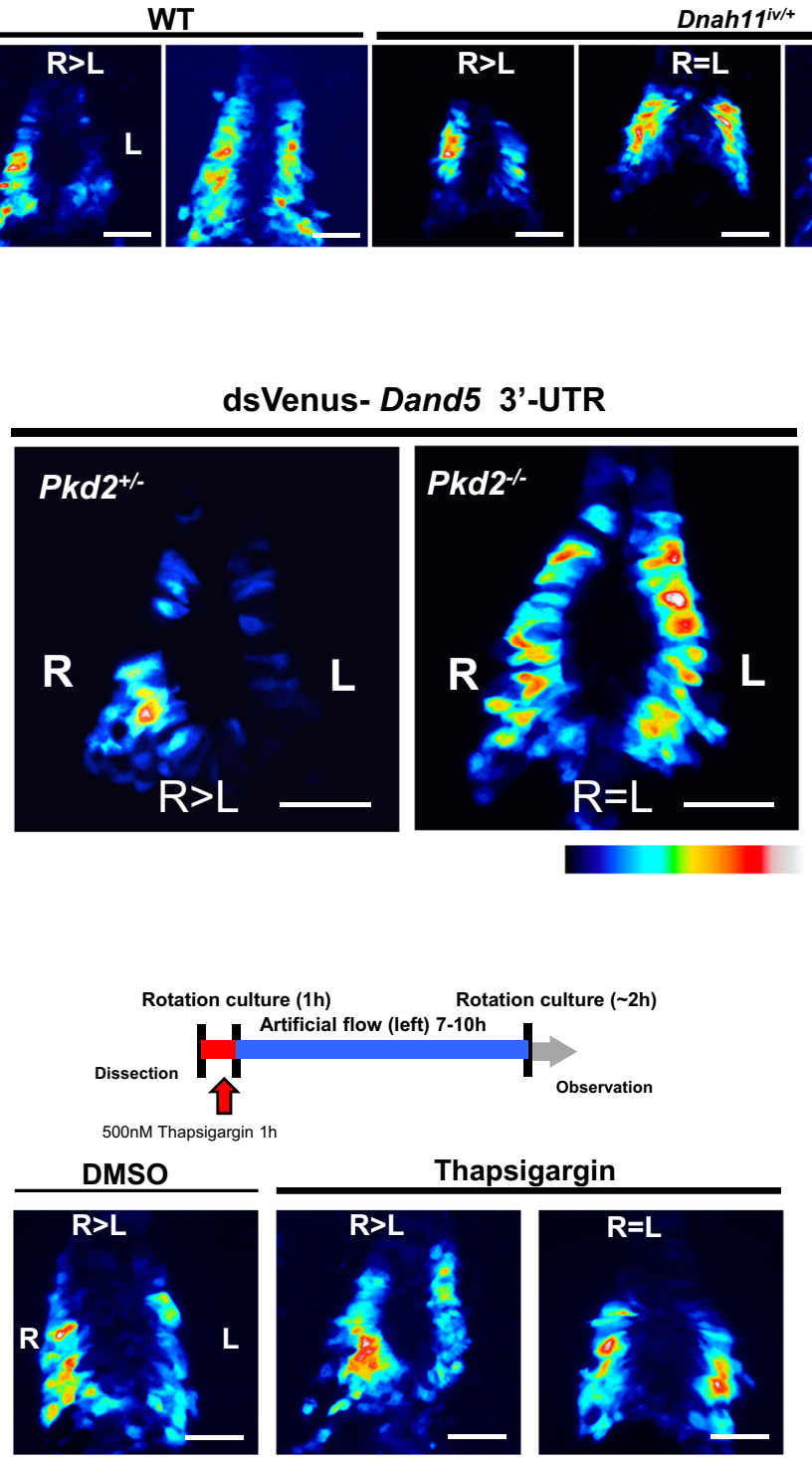

b

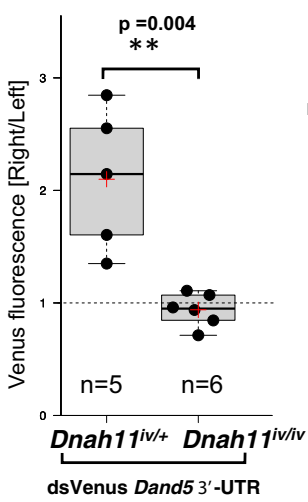

C

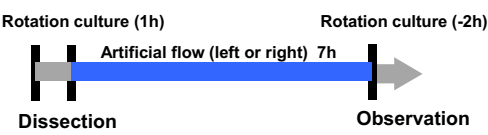

Artificial $<$ Endogenous

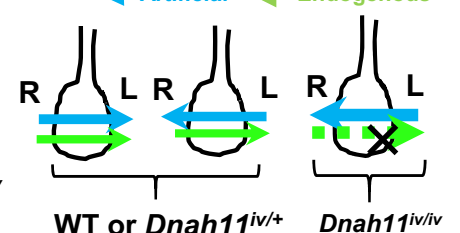

WT or Dnah11 1iv/+ Dnah11 1iv/v

Artificial rightward flow
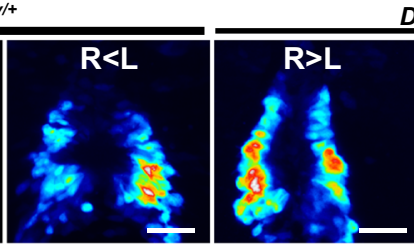

Dnah11iviv

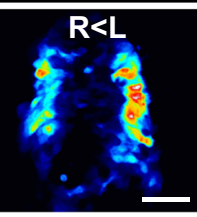

+1
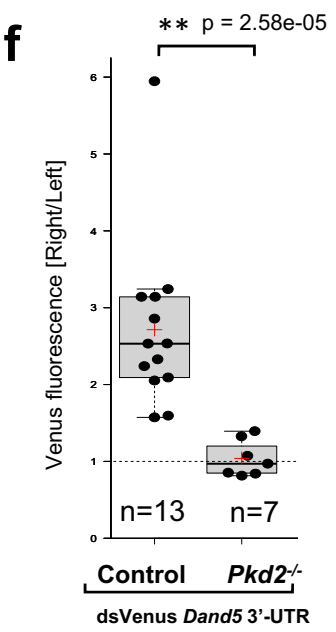

h

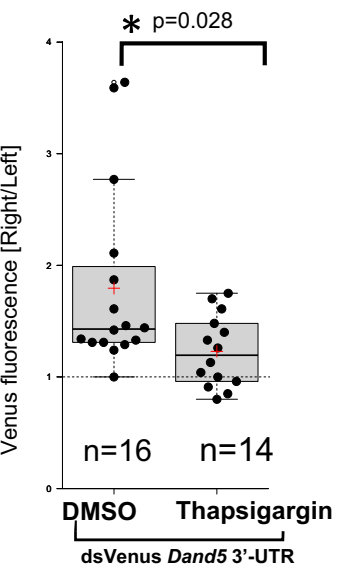

examined, one embryo lost Nodal expression in LPM, suggesting that the Dand $5^{\Delta 200 \text { prox }}$ allele may act dominantly in rare instances. WISH analysis also showed that Dand5 mRNA was not distributed asymmetrically in Dand $5^{\Delta 200 \text { prox/ }} \Delta 200$ prox embryos $(n$ $=5 / 5$ ) (Fig. 3d). Together, these results indicated that the 200 nucleotide proximal region of the $3^{\prime}$-UTR is involved in the L-R asymmetric pattern of Dand5 mRNA in node crown cells.

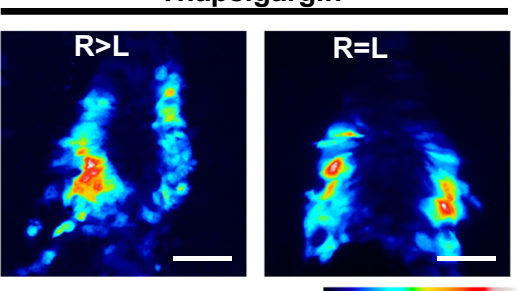

Flow-induced decay of Dand5 mRNA depends on Bicc1. We hypothesized that a specific factor (or factors) in crown cells binds to the proximal portion of the $3^{\prime}$-UTR to mediate the decay of Dand5 mRNA preferentially on the left side of the node. Among candidates for such a factor, we focused on Biccl, an RNA binding protein with $\mathrm{KH}$ domains for RNA binding and a SAM domain for polymerization ${ }^{12,23}$, given that it is specifically 
Fig. 2 The 3'-UTR of Dand5 mRNA responds to the direction of fluid flow in a Pkd2-dependent manner. a Fluorescence of dsVenus at the node of Dnah11iv/+ or Dnah17iv/iv embryos harboring the NDE-Hsp-dsVenus-3'-UTR transgene at the five-somite stage. The color scale indicates the intensity of ds Venus fluorescence, with white corresponding to the maximal level. Images shown are representative of at least five embryos for each genotype. b Ratios of fluorescence on the right versus left side of the node at four- to six-somite stages shown for each genotype. The $n$ values indicate the numbers of embryos analyzed. Red crosses indicate average values. c Design of artificial flow experiments. Dnah11+/+ (WT), Dnah11 ${ }^{\mathrm{iv} /+}$, or Dnah17iv/iv embryos harboring the NDE-Hsp-dsVenus-3'-UTR transgene were cultured under the influence of a rightward or leftward artificial flow from the late headfold to fivesomite stages. d Fluorescence of ds Venus at the node of representative embryos of the indicated genotypes after exposure to the leftward or rightward artificial flow as in (c). e, f Fluorescence of dsVenus at the node of NDE-Hsp-dsVenus-3'-UTR transgenic Pkd2 ${ }^{+/-}$or Pkd2 ${ }^{-/-}$embryos at the four- to sixsomite stage (e) and its R/L ratios (f) quantified as in (b). $\mathbf{g}$ DsVenus fluorescence at the node of NDE-Hsp-dsVenus-3'-UTR embryos that were incubated at the four- to six-somite stage with thapsigargin $(500 \mathrm{nM})$ or dimethyl sulfoxide (DMSO, vehicle), followed by administration of a leftward artificial flow. Scale bars, $50 \mu \mathrm{m}$. h Right/Left ratios of dsVenus fluorescence in groups of DMSO-treated versus thapsigargin-treated embryos from the experiment in ( $\mathbf{g}$ ) plotted as in (b) and (f). All scale bars, $50 \mu \mathrm{m}$. For the boxplots in (b, $\mathbf{f}$, and $\mathbf{h}$ ), the top and bottom lines of each box represent the 75th and 25th percentiles of the samples, respectively. The line inside each box represents the median of the samples. The upper and lower lines above and below the boxes are the whiskers. Statistical significance $(p)$ was determined using the two-sided Wilcoxon rank sum test. Source data are provided as a Source Data file.

expressed at the node of mouse embryos and that Bicc1 $1^{-/-}$ embryos manifest laterality defects ${ }^{10}$. Whole-mount immunofluorescence analysis revealed that pit cells and crown cells at the node express Bicc1 in an apparently L-R symmetric manner (Fig. $4 \mathrm{a}-\mathrm{c}$ ). Moreover, whereas Dand5 mRNA is localized predominantly at the apical side of crown cells ${ }^{24}$, Biccl was detected both apically and basally (Fig. 4d). To evaluate potential genetic interaction between Bicc1 and Dand5, we deleted the first exon of Bicc1 using the CRISPR/Cas9 system (Supplementary Fig. 4a). The fluorescence of dsVenus in the resulting Bicc $1^{\Delta \operatorname{ex} 1 / \Delta \operatorname{ex} 1}$ embryos harboring the NDE-Hsp-dsVenus-3'-UTR transgene was bilaterally symmetric $(n=6 / 6)$ (Fig. 4e, Supplementary Fig. 1c), suggesting that the decay of Dand5 mRNA on the left side is impaired in the absence of Biccl. Similar to our previous observations with $\mathrm{Bicc1}^{-/-}$embryos $^{10}$, the basal body of motile cilia at the node failed to shift posteriorly in half of the $B i c c 1^{\Delta \operatorname{ex} 1 / \Delta \operatorname{exl}}$ embryos examined $(n=3 / 6)$ (Supplementary Fig. $4 \mathrm{~b}$ and c). Consistent with this finding, the leftward flow at the node was disrupted in half of $B i c c 1^{\Delta \operatorname{ex} 1 / \Delta \text { exl }}$ embryos $(n=5 / 9)$, with the other half ( $n=4 / 9$ embryos) showing normal leftward flow (Fig. 4f). Nonetheless, an artificial leftward fluid flow failed to rescue the pattern of $\mathrm{dsVenus}$ fluorescence in $B i c c 1^{\Delta \operatorname{ex} 1 / \Delta \operatorname{exl}}$ embryos examined $(n=7 / 7)$, suggesting that the mutant embryos are unable to respond to the fluid flow (Fig. $4 \mathrm{~g}$ and $\mathrm{h}$ ).

Biccl binds to the $3^{\prime}$-UTR of Dand5 mRNA. To assess whether the 200-nucleotide proximal portion of the $3^{\prime}$-UTR of Dand5 mRNA mediates the binding of Biccl, we ligated dsVenus $-3^{\prime}$ UTR, dsVenus-3'-UTRA200prox or dsVenus-3'-UTR(200prox) mRNA to a ubiquitous promoter for co-expression with hemagglutinin epitope (HA)-tagged mouse Biccl in HEK293T cells. Reverse transcription (RT) and quantitative polymerase chain reaction (qPCR) analysis of anti-HA immunoprecipitates and input extracts revealed that the $d s$ Venus $-3^{\prime}$ UTR and dsVenus-3'-UTR(200prox) mRNAs were enriched in the HA-Bicc1 precipitates (Fig. 5a-e). By contrast, a truncated form of Bicc1 lacking all $\mathrm{KH}$ domains $(\Delta \mathrm{KH})^{10}$ failed to recruit the WT dsVenus-3'-UTR mRNA (Fig. 5b and c). In KH domains, a conserved GXXG motif (where at least one $\mathrm{X}$ is a basic residue) binds single-stranded RNA sequences of 4 nucleotides and orients them toward an adjacent groove for nucleobase recognition ${ }^{11,23}$ (Fig. 5f). To evaluate the contribution of individual GXXG motifs to RNA binding, we mutated them to GDDG ${ }^{25}$. Mutation of the $\mathrm{KH} 1$ domain reduced the binding of Biccl to the $d s$ Venus- $3^{\prime}-U T R$ reporter mRNA more than twofold, and mutation of the $\mathrm{KH} 2$ domain or of all three $\mathrm{KH}$ domains together nearly abolished it. By contrast, mutation of the $\mathrm{KH} 3$ domain alone had no significant effect on binding (Fig. $5 \mathrm{~g}$ ). Together, these results suggest that binding of Biccl to the $3^{\prime}$-UTR of Dand5 mRNA is mediated by the 200-nucleotide proximal portion of the $3^{\prime}$-UTR and the $\mathrm{KH} 1$ and $\mathrm{KH} 2$ domains of Bicc1. However, binding to HA-Bicc1 alone did not reduce $d s$ Venus-3'-UTR reporter mRNA translation (Fig. 5b, anti-GFP blot), indicating that the regulation of Bicc1 activity by nodal flow in vivo requires additional factors that are absent or inactive in HEK293T cells.

Bicc1 specifically recognizes a conserved 8-mer motif in the Dand5 3'-UTR. To identify where Biccl binds the $3^{\prime}$-UTR of Dand5 mRNA, we performed RNA Bind-n-Seq (RBNS) analysis $^{26}$ with a random 20-mer RNA library using lysates of 293FT cells expressing FLAG-tagged Bicc1 (Fig. 6a). Analysis of Bicc1-interacting RNA 20-mers showed that the GAC-containing motifs were highly enriched (Fig. 6b and c), with YGAC (where Y represents a pyrimidine) and GACR (where R represents a purine) motifs showing higher enrichment scores than other GAC motifs (Fig. 6d). Both in mammalian and Xenopus Dand5 3'UTRs, GACR and YGAC motifs were most enriched in the 200nucleotide proximal regions (Fig. 6e). Metagene analysis with the 200-nucleotide proximal region of the $3^{\prime}$-UTR of mouse mRNAs revealed that the frequency of GACR and GAC was significantly enriched in the Dand5 $3^{\prime}$-UTR (Fig. $6 f$ and g). Furthermore, among 8-mer sequences, GACN ${ }_{1-2}$ GAC sequences containing two GAC motifs were also enriched (Supplementary Fig. 5), suggesting a preference of Biccl for GACR and YGAC motifs alone or in combination. The juxtaposed GACR and YGAC motifs appear to be conserved in mammals, and a similar bipartite motif(s) is found in Xenopus and medaka fish. Thus, the Xenopus sequence CGACUUGAC at this position corresponds to two YGAC motifs, followed by the UGACCGACG in close proximity (Fig. 6h, Supplementary Fig. 6). The consensus motif $\mathrm{GACN}_{1-3} \mathrm{GAC}$ thus appeared as a likely candidate to mediate the recruitment of mammalian Bicc1 (Fig. 6h). Zebrafish Dand5 mRNA and other target mRNAs of Biccl lack such a bipartite motif, although they contain GAC motifs in their $3^{\prime}$-UTRs (Supplementary Fig. 6). Bicc1 may recognize different panels of sequences by using distinct combinations of its three $\mathrm{KH}$ domains.

Secondary structure prediction based on sequence conservation revealed that the $3^{\prime}$ half of this GACGUGAC motif (UGAC in murine Dand5 $3^{\prime}$-UTR) may be buried in a stable hairpin (Supplementary Fig. 7a). To assess Bicc1 binding, 45- to 225nucleotide RNA probes comprising this motif were fluorescently labeled and incubated in vitro with increasing concentrations of recombinant GST-KH domains (Supplementary Fig. 7b). Analysis by Electrophoretic Mobility Shift Assays (EMSA) revealed that GST-KH shifted all three probes into several ribonucleoprotein 


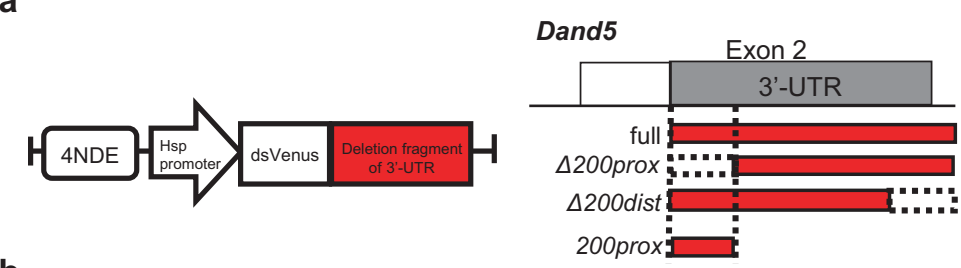

b

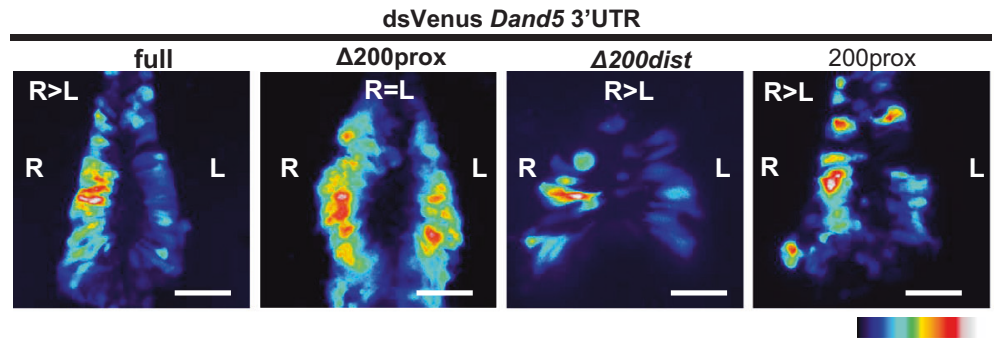

C
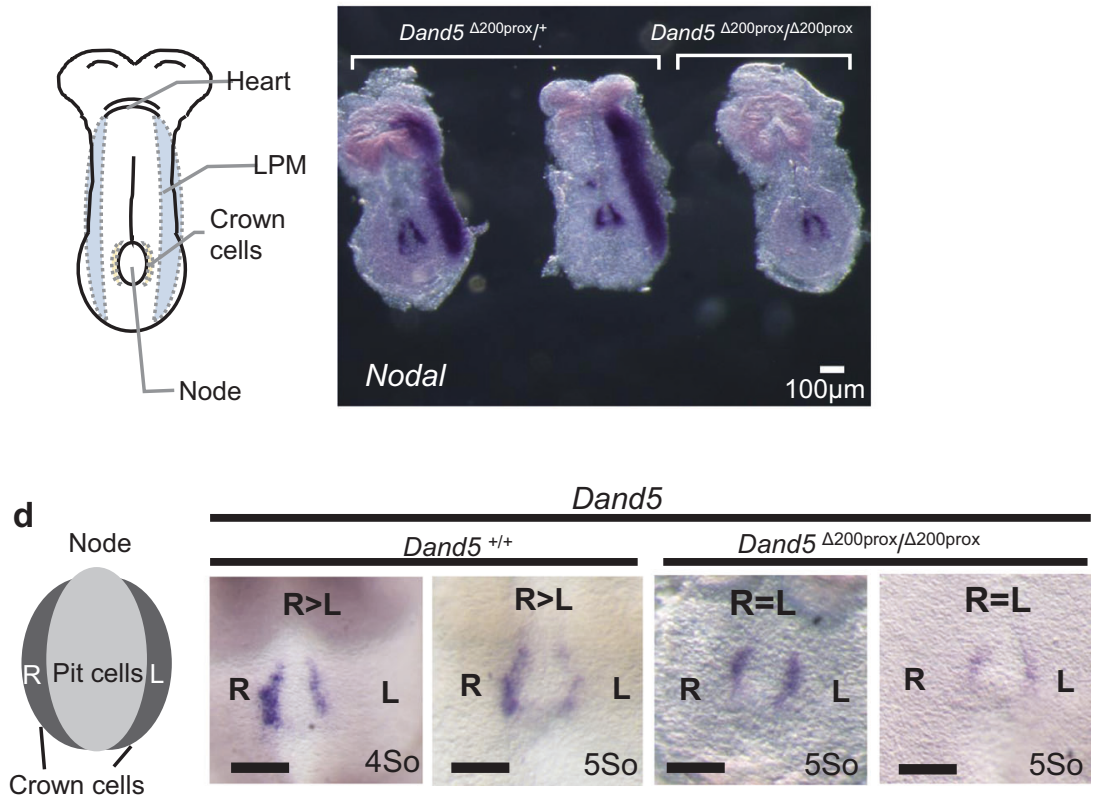

Fig. 3 A 200-nucleotide conserved proximal region of the $3^{\prime}$-UTR is required for L-R asymmetric accumulation of Dand5 mRNA. a Schematic representation of the NDE-Hsp-dsVenus transgene fused to the Dand5 3'-UTR (left) or to a series of various 3'-UTR fragments (right). b Fluorescence of dsVenus at the node of four- to six-somite stage mouse embryos harboring the indicated transgene (see also Supplementary Fig. 1b). Images are representatives of at least three embryos for each transgene. Scale bar, $50 \mu \mathrm{m}$. c WISH analysis of Nodal expression in Dand5 $5200 p r o x /+$ and

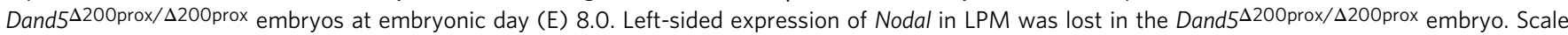
bar, $100 \mu \mathrm{m}$. Images are representatives of at least four embryos for each genotype. d WISH analysis of Dand5 expression at the node of Dand5 $+/+$ and Dand5 $\Delta 200$ prox/ $\Delta 200$ prox embryos at four- to five-somite stages (E8.0). Two representative embryos are shown for each genotype. L-R asymmetry of Dand5 mRNA at the node was lost in the Dand5 200 prox/ 200 prox embryo. Scale bars, $100 \mu \mathrm{m}$.

(RNP) complexes (Supplementary Fig. 7c), with the highest affinity complex $\mathrm{RNP}_{1}$ showing an apparent $\mathrm{Kd}$ of $233 \mathrm{nM}$ for the 225 -nucleotide probe, compared to only $120 \mathrm{nM}$ for the shorter 35-157 and 66-110 probes, respectively. Additional RNPs migrating with slower mobility only formed at elevated GST$\mathrm{KH}$ concentrations with all three RNA probes, suggesting they likely represent higher-order complexes. To assess specificity, we performed competition experiments by incubating pre-assembled RNPs with increasing amounts of non-fluorescent competitor transcripts. The half maximal inhibitory concentration (IC50) of unlabeled Dand5 3'-UTR 1-225 RNA was observed at $283 \mathrm{nM}$, whereas the distal Dand5 $3^{\prime}-U T R_{226-425}$ fragment, which is similar in size but contains no GAC motifs, was not competitive (IC50 > $1 \mu \mathrm{M}$ ) (Supplementary Fig. 7b and d). Similarly, unlabeled 35-157 and 66-110 fragments competed with fluorescent probes, showing IC50 values of $191 \mathrm{nM}$ and $203 \mathrm{nM}$ respectively, whereas control fragments of similar size from the distal $3^{\prime}$-UTR did not (Supplementary Fig. 7e and f). These results confirm that the proximal segment of the Dand5 $3^{\prime}-U T R$ has a higher affinity for Biccl than the distal region.

To evaluate the contribution of the GACGUGAC motif to Biccl binding, we tested the influence of specific mutations in the short Dand5 3'-UTR $66-110$ fragment (Fig. 7a). Analysis of the 3'$U R_{66-110} M u t 1$ RNA probe by competitive EMSA showed that mutation of GACG to AAGG abolished competitive GST-KH binding (IC50 $>1 \mu \mathrm{M})$ (Fig. 7b). Likewise, the $3^{\prime}-U T R_{66-110}$ Stem + probe that was engineered to mask the GACG motif in an elongated stem, lacked competitive binding activity (IC50 > 

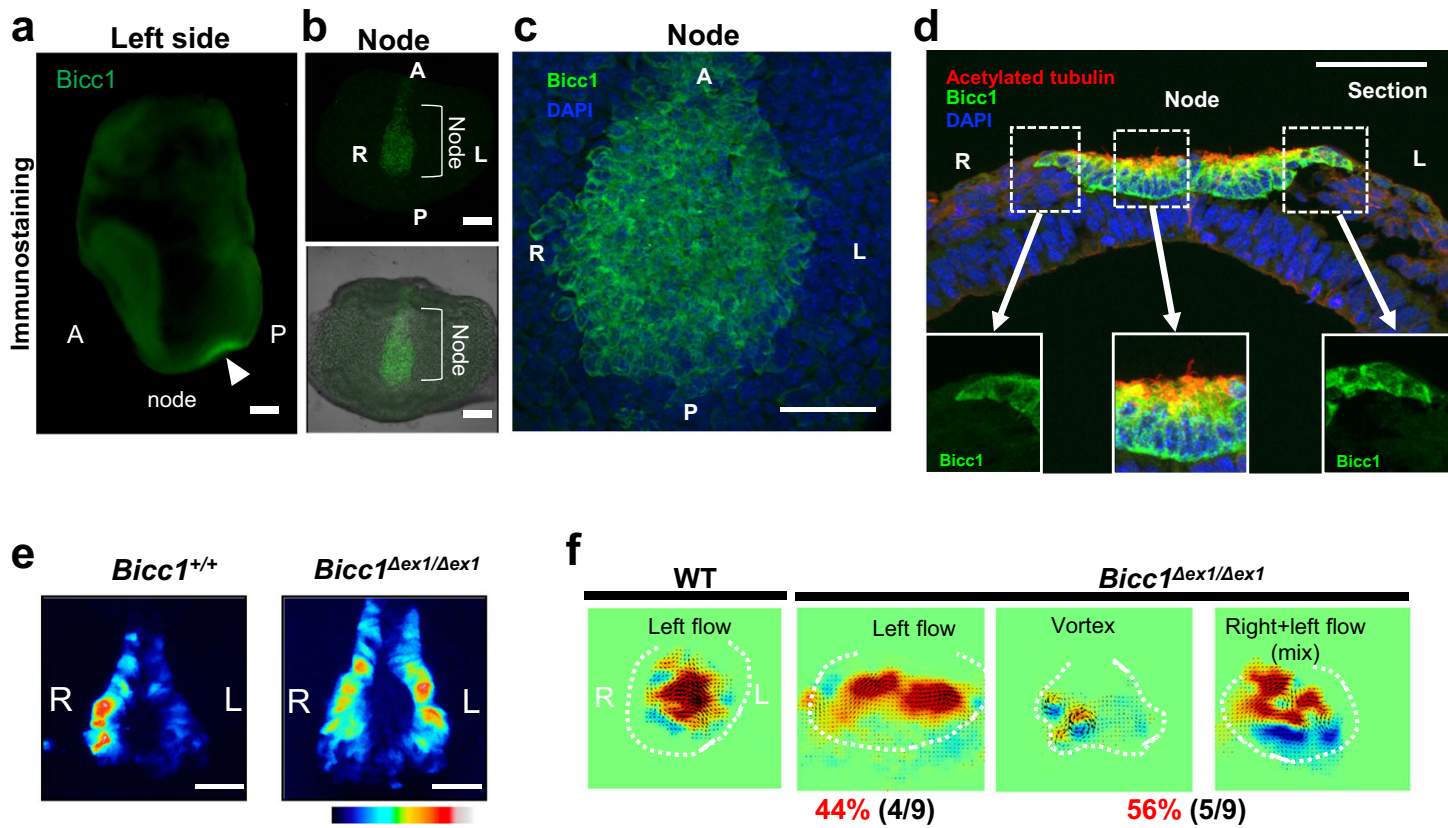

f

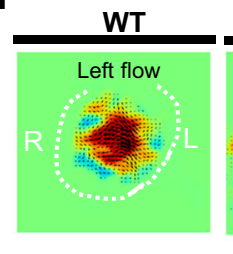

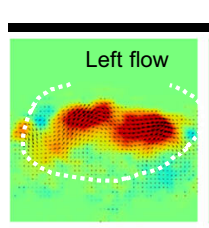

$44 \%(4 / 9)$

\section{$\operatorname{Bicc1}^{\Delta e \times 1 / \Delta e x 1}$}

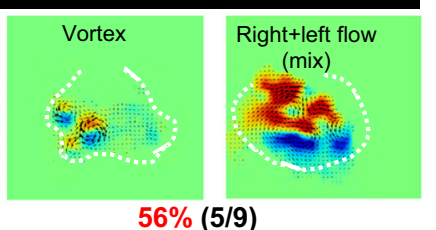

h

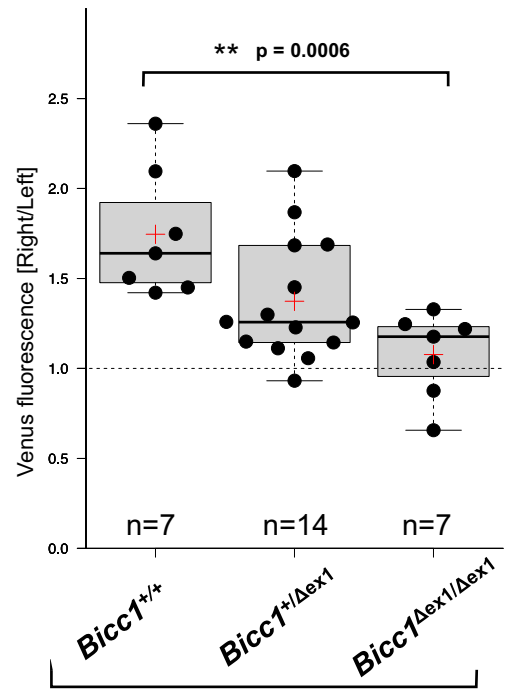

dsVenus Dand5 3'-UTR g

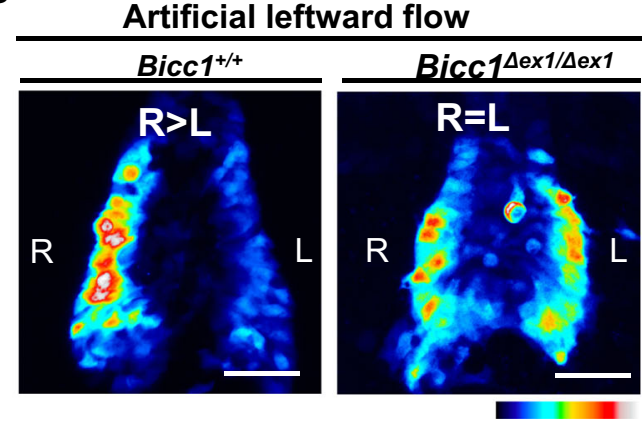

Artificial leftward flow

Fig. 4 Flow-induced decay of Dand5 mRNA depends on Bicc1. a-d Immunofluorescence staining of Bicc1 in node cells of a WT embryo at E8.0 (a), or its node region at higher magnification (b, c), and in a transverse section of the node (d). Nuclei were stained with 4',6-diamidino-2-phenylindole (DAPI) in (c) and (d), and acetylated tubulin was also immunostained in (d) to reveal cilia. Scale bars in (a, b) and (c, d) are $100 \mu \mathrm{m}$ and $50 \mu \mathrm{m}$, respectively. Images shown in (a-d) are representative of at least two independent experiments. e dsVenus fluorescence at the node of four- to six-somite stage WT and Bicc ${ }^{\Delta \mathrm{ex} 1 / \Delta \mathrm{ex} 1}$ embryos harboring the NDE-Hsp-dsVenus-3'-UTR transgene. Scale bars, $50 \mu \mathrm{m}$. Images shown are representative of six embryos for each genotype. $\mathbf{f}$ Nodal flow in WT and Bicc ${ }^{\Delta \mathrm{ex}} 1 / \Delta \mathrm{ex} 1$ mouse embryos at three- to five-somite stages as revealed by particle image velocimetry (PIV) analysis. The dashed white line indicates the outline of the node, small arrows in the node region indicate the direction and velocity of flow at those positions. The relative color scale indicates the magnitude of flow velocity (leftward in red, rightward in blue). Note that leftward laminar flow was lost in five out of nine

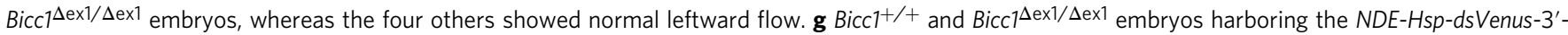
UTR transgene were cultured in the presence of an artificial leftward flow as in Fig. 2c, after which the L-R pattern of dsVenus fluorescence at the node was examined. Scale bars, $50 \mu \mathrm{m}$. h Quantification of Right/Left ratios of dsVenus fluorescence at the node of NDE-Hsp-dsVenus-3'-UTR embryos of the indicated genotypes after culture in leftward artificial flow as in $(\mathbf{g})$. The $\mathrm{n}$ values indicate the numbers of embryos analyzed, and red crosses indicate average values. For the boxplots, the top and bottom lines of each box represent the 75th and 25th percentiles of the samples, respectively. The line inside each box represents the median of the samples. The upper and lower lines above and below the boxes are the whiskers. Statistical significance ( $p$ ) was determined using the two-sided Wilcoxon rank sum test. Source data are provided as a Source Data file. Note that the artificial leftward fluid flow failed to rescue the impaired L-R pattern of dsVenus fluorescence in the Bicc ${ }^{\Delta \mathrm{ex} 1 / \Delta \mathrm{ex} 1}$ embryos. 


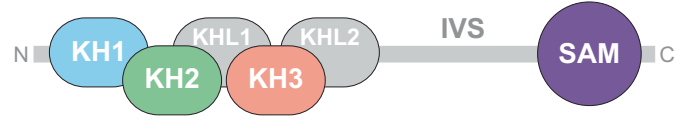

b

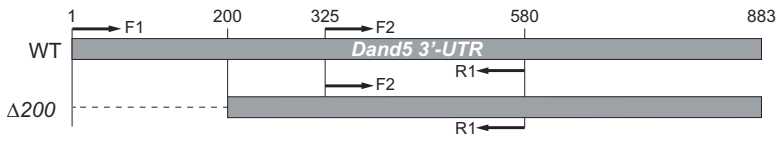

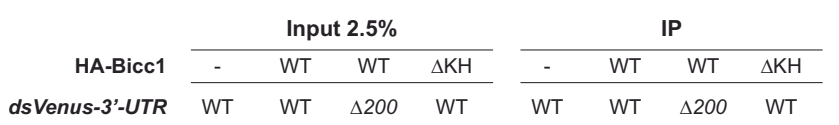

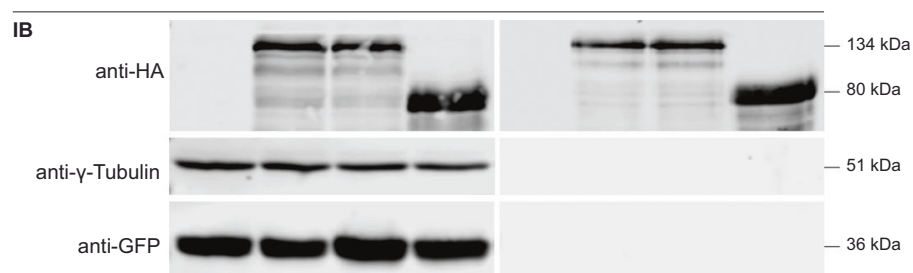

RT-PCR

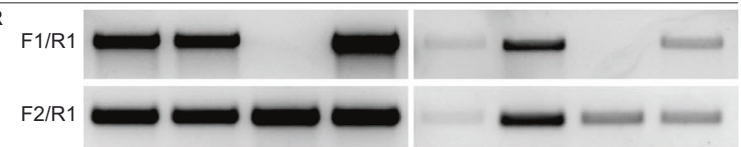

d

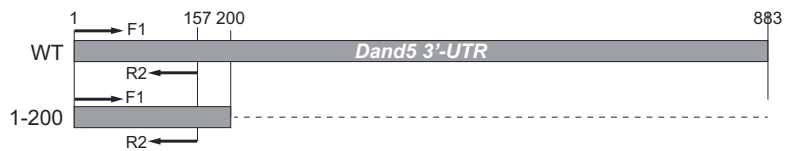

\begin{tabular}{rlllllll} 
& \multicolumn{3}{c}{ Input 2.5\% } & & \multicolumn{3}{c}{ IP } \\
\cline { 2 - 4 } \cline { 5 - 6 } HA-Bicc1 & - & WT & WT & & - & WT & WT \\
\cline { 2 - 7 } dsVenus-3'-UTR & WT & WT & $1-200$ & & WT & WT & $1-200$
\end{tabular}

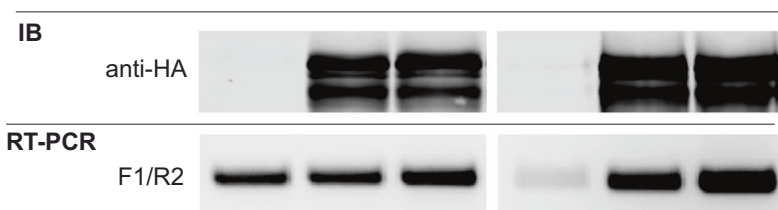

f

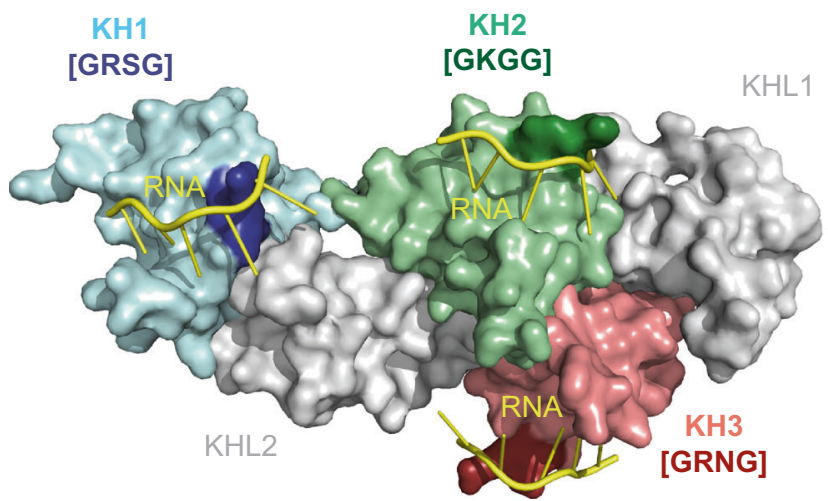

$1 \mu \mathrm{M})$. Competitive binding also sharply decreased upon mutation of UGAC to UAAG, as shown by the $3^{\prime}-$ UTR $_{66-110} M u t 2$ probe $(\mathrm{IC} 50=654 \mathrm{nM})$. To rule out a non-specific inhibitory effect resulting from disruption of the stem, we generated $3^{\prime}$ UTR $_{66-110} \mathrm{Mut} 2^{\text {Stem }}$ where a UAAG-complementary sequence should restore stem-loop formation (Fig. 7a). No difference was
C

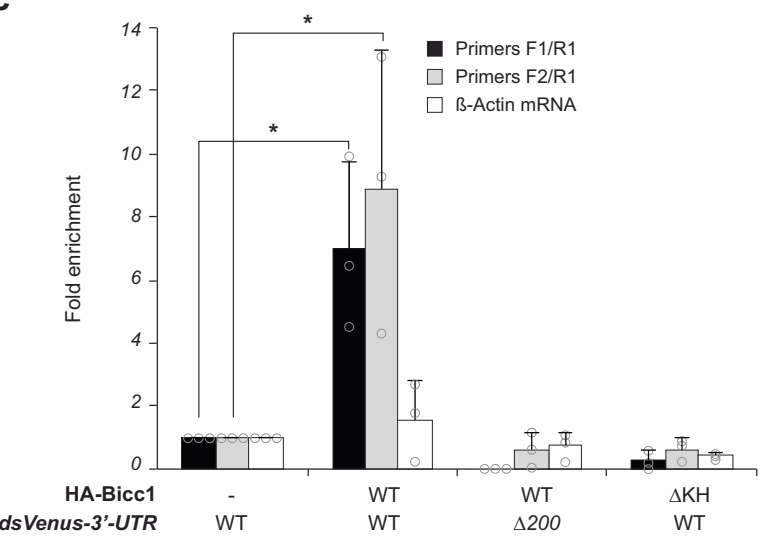

e
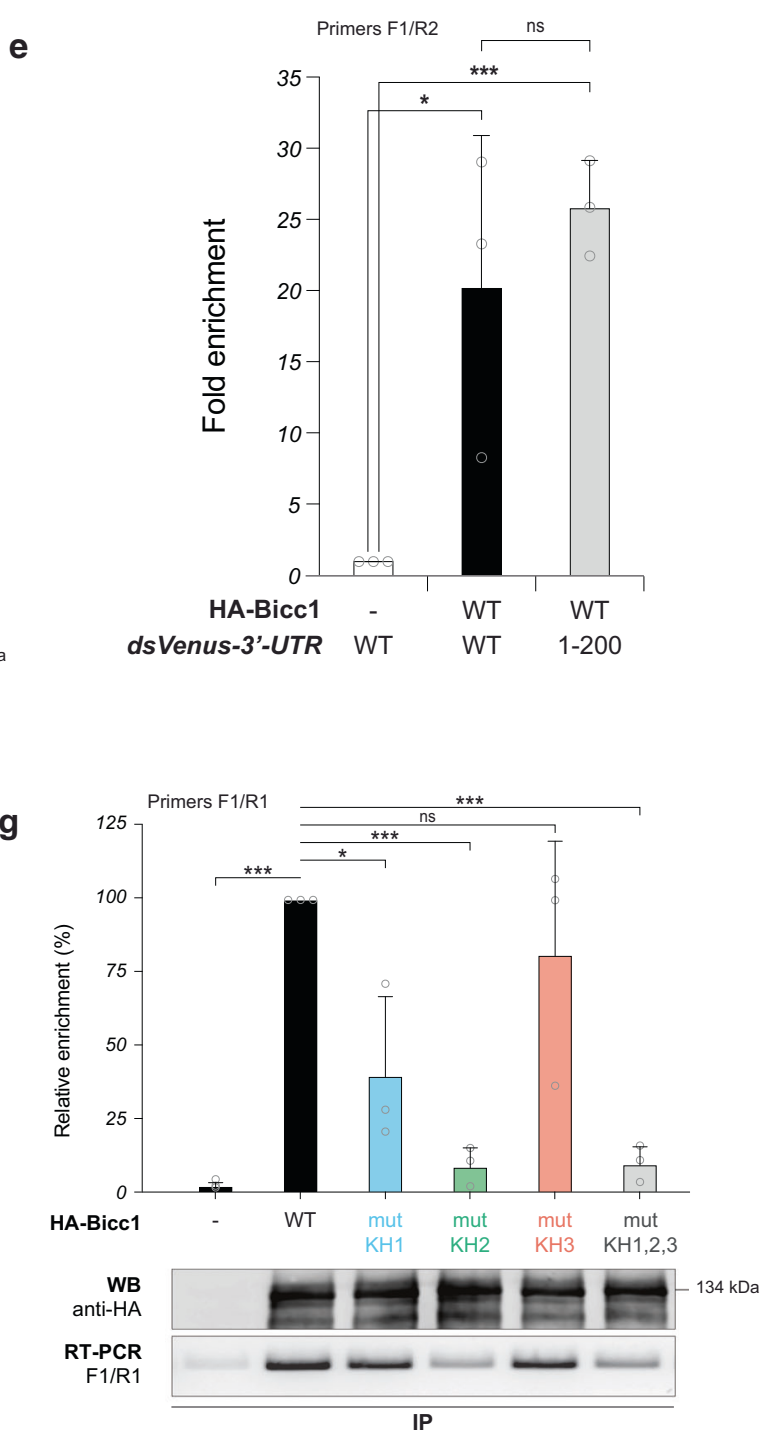

observed between these two mutants in their affinity for Bicc1, suggesting that specific Biccl binding depends on the GACGUGAC sequence and not just on the secondary structure (Fig. 7b, bottom panels). As another independent specificity control, we used the GACGUGAC octamer to replace a similar, though nonspecific, GCCCUGGG sequence after two adenosines in the distal 
Fig. 5 Bicc1 binds to the proximal 3'-UTR of Dand5 mRNA via its KH1 and KH2 domains. a Domain organization of Bicc1. KH: K homology; $\mathrm{KHL}$ : KH-like; IVS: Intervening sequence; SAM: Sterile $\alpha$ motif. b Co-immunoprecipitation of mRNAs with HA-Bicc1 in HEK293T cells, and PCR primers used to detect Dand5 reporter mRNAs by RT-PCR or -qPCR analysis. The primers F1/R1 detect only dsVenus-3'-UTR mRNA, whereas the F2/R1 primers also detect dsVenus-3'-UTRA200prox. Extracts of cells co-expressing the indicated reporter constructs and HA-Bicc1 (WT) or HA-Bicc1 $\triangle K H$ were subjected to anti-HA immunoprecipitation. Immunoprecipitates (IP) and portions of the input extracts were subjected to anti-HA Western blot (WB) and to RT-PCR analysis. c RT-qPCR analysis of immunoprecipitates as in (b), but including $\beta$-actin mRNA (negative control). Data represent the ratio of co-immunoprecipitated mRNA to the input relative to the corresponding condition without HA-Bicc1. Source data are provided as a Source Data file. $\mathbf{d}$ Co-immunoprecipitation of reporter mRNAs with HA-Bicc1 in HEK293T cells. Extracts of cells expressing the indicated constructs were prepared as in (b). e RT-qPCR analysis of immunoprecipitates from the experiments in (d). Results are expressed as the ratio of co-immunoprecipitated mRNA to the input, normalized to the corresponding condition without HA-Bicc1. Source data are provided as a Source Data file. $\mathbf{f}$ Three-dimensional model of the mouse Bicc1 KH domain repeat in surface representation. Putative RNA-binding GXXG motifs (where at least one X is a basic residue) are highlighted. $\mathbf{g}$ RT-qPCR and Western blot analyses of immunoprecipitates prepared as in (c) from HEK293T cells expressing the dsVenus-3'-UTR construct and wild-type HA-Bicc1 (WT) or mutant versions in which the GXXG motif of individual $\mathrm{KH}$ domains (mutKH1, mutKH2, or mutKH3) or all three $\mathrm{KH}$ domains (mutKH1,2,3) were replaced with GDDG. RT-qPCR data represent the ratio of co-immunoprecipitated mRNA to the input and are expressed as a percentage of the value for HA-Bicc1(WT). Data in (c), (e) and (g) are means + SD from three independent experiments. ${ }^{\star} p<0.05,{ }^{\star \star} p<0.01,{ }^{\star \star \star} p<0.001$ (two-sided Student's $t$-test). Source data are provided as a Source Data file.

226-270 fragment (Supplementary Fig. 8a). Interestingly, the resulting $3^{\prime}-U T R_{226-270} 8$-mer RNA probe competed for Bicc1 KH domains with high affinity $(\mathrm{IC} 50=392 \mathrm{nM})$, indicating that one GACGUGAC sequence is sufficient to confer specific binding (Supplementary Fig. 8b).

To directly test the role of the GACGUGAC motif in the dsVenus-3'-UTR reporter, we replaced critical nucleotides by uridines (UAUGUUAU). Interestingly, the resulting mutU reporter mRNA failed to bind Biccl above background levels (Fig. 7c). Similarly, the $3^{\prime} \mathrm{UTR}_{86-110}$ Stem + reporter which was designed to mask the GACGUGAC octamer in an elongated stem failed to coimmunoprecipitate with HA-Biccl (Fig. 7c), confirming the loss of binding observed in competitive EMSA assays (Fig. 7b). Together, these results indicate that the specific recruitment of Biccl to the Dand5 $3^{\prime}-U T R$ is mediated by the bipartite GACGUGAC motif. The juxtaposition of GACG and UGAC within this motif likely explains why its specific recognition by Bicc1 involves both the $\mathrm{KH} 2$ and the $\mathrm{KH} 1$ domains (Supplementary Fig. 9).

Decay of Dand5 mRNA on the left side of the node requires the Ccr4-not deadenylase complex. In Drosophila, an ortholog of Bicc1 regulates mRNA decay by recruiting the Ccr4-Not complex through direct binding to its Not $3 / 5$ subunit $^{15}$. Although Bicc1mediated mRNA decay has not been observed previously in vertebrates $^{11,27}$, several Ccr4-Not subunits were also found to be enriched by mouse Biccl in a protein interactome screen in $\mathrm{T}$ Rex HEK293 cells ${ }^{28}$. Consistent with a possible interaction in vivo, immunofluorescent staining of endogenous Biccl and Cnot3 proteins in mouse embryos significantly overlapped in the cytoplasm of node crown cells (Fig. 8a). In good agreement, immunostaining of transfected Cnot3-FLAG overlapped with large cytoplasmic HA-Biccl foci at their periphery, and with polymerization mutant HA-Biccl(mutD), which stains more diffusely due to a mutation in the SAM-SAM interface (Supplementary Fig. 10). To further test this interaction and whether it involves Biccl polymerization or RNA binding, we performed coimmunoprecipitation assays in HEK293T cells. Both endogenous Cnot1 and FLAG-tagged Cnot3 co-immunoprecipitated with HA-Bicc1, irrespective of prior treatment with RNase A (Fig. $8 \mathrm{~b}$ and c). Moreover, while deletion of the Bicc1 KH repeats significantly diminished the co-immunoprecipitation of both Cnot3-Flag and of endogenous Cnot1, deletion of the SAM domain did not (in the case of Cnot1) or even increased it (in the case of Cnot3-FLAG). Besides corroborating the observed protein colocalization, these results suggest that mammalian Bicc1 recruits the CCR4-Not complex at least in part through its $\mathrm{KH}$ domains, independently of RNA and of the SAM domain.

To examine whether Cnot 3 contributes to the decay of Dand5 mRNA in crown cells at the node, we generated Cnot3 conditional knockout (CKO) embryos by crossing a Cnot3 $3^{\text {flox/ }}$ flox mouse ${ }^{29}$ with a Noto ${ }^{\text {CreERT2/+ }}$ mouse ${ }^{30}$. The latter expresses Cre recombinase in a tamoxifen-inducible manner specifically at the node beginning at E8.0. Control (Cnot3 $3^{\text {flox/flox; }}$ Noto ${ }^{+1+}$, Cnot $3^{\text {flox } /+} ;$ Noto $^{+/+}$, Cnot $3^{+/+} ;$Noto $^{\text {CreERT } 2 /+}$ and Cnot $3^{+/+}$; Noto CreERT2/CreERT2) and CKO (Cnot $3^{\text {flox/flox;NNoto CreERT2/+ or }}$ CreERT2/CreERT2) embryos isolated from tamoxifen-treated dams were first examined for Nodal expression. Nodal expression was maintained at the node and in LPM in all the control embryos examined $(n=8 / 8)$ (Fig. $8 d)$. In about a half of Cnot3 CKO embryos $(n=7 / 13)$, Nodal mRNA was maintained at the node but was lost in LPM (Fig. 8d), consistent with the possibility that the stability of Dand5 mRNA in crown cells was increased. We then examined Dand5 expression in control and CKO embryos at the four- to six-somite stages. L-R asymmetry of Dand5 mRNA was lost $(n=6 / 14)$ or attenuated $(n=6 / 14)$ in the CKO embryos Fig. 8e, g). Similarly, L-R asymmetry of the $3^{\prime}$-UTR reporter transgene was lost or attenuated in the CKO embryos $(n=7 / 7)$ (Fig. 8f, and Supplementary Fig. 1d). When Cnot3 CKO and control embryos were examined at a later stage (E9.5), some of the CKO embryos (2/25 embryos) exhibited heart looping defects whereas none of the control embryos (0/25 embryos) showed such heart defects (Supplementary Fig. 11). The low frequency of the heart defects in the CKO embryos is likely due to the incomplete deletion by Noto ${ }^{C r e E R T 2}$ and the robustness of the asymmetry-generating system that operates at the node and in the $\mathrm{LPM}^{24,31}$. These results suggest that the Ccr4-Not complex in crown cells indeed plays a role in the degradation of Dand5 mRNA in response to nodal flow (Fig. 9).

\section{Discussion}

To delineate how fluid flow establishes left-right asymmetry in the mouse embryo, we have generated a dsVenus transgene that reports the decay of Dand5 mRNA in crown cells of the node and its acceleration by directional fluid flow and by Pkd2 specifically on the left side. Our data show that a proximal $3^{\prime}$-UTR fragment mediating asymmetric Dand5 mRNA expression binds and genetically interacts with the RNA binding protein Biccl through a newly identified Bicc1-binding element comprising two GAC motifs that are juxtaposed within the octameric sequence GACGUGAC. In turn, Biccl binds to the Ccr4-Not deadenylase complex which mediates increased 
a

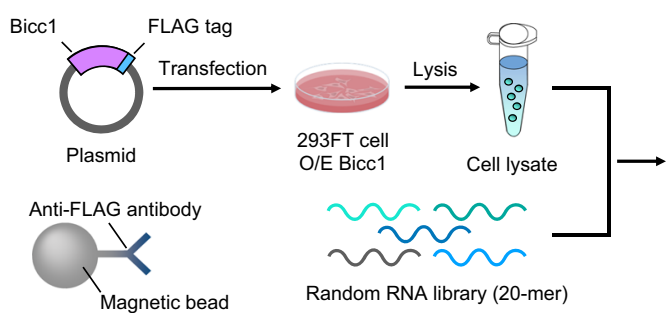

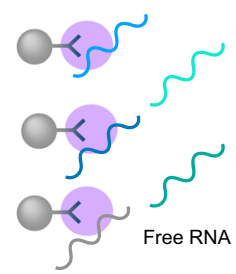

Bicc1 binding RNA

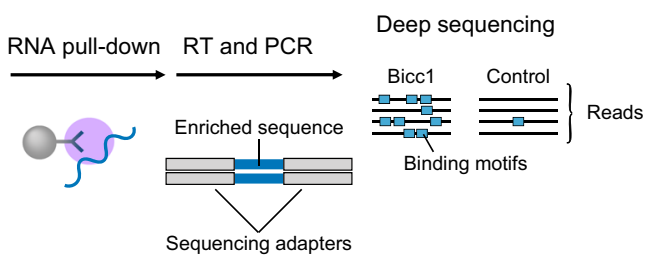

b

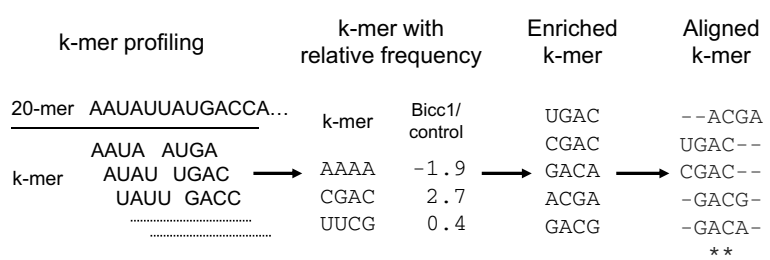

C

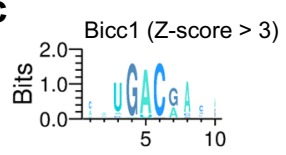

d
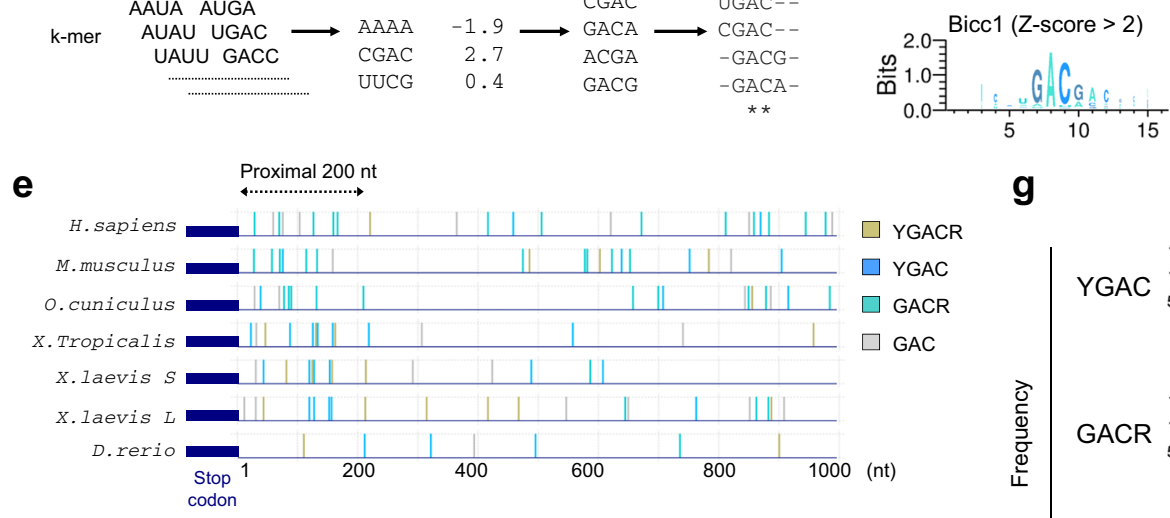

\begin{tabular}{|c|c|c|c|}
\hline Rank & 4-mer & 5-mer & 6 -mer \\
\hline 1 & UGAC & GACGA & UGACGA \\
\hline 2 & CGAC & ACGAC & UGACAA \\
\hline 3 & GACA & AUGAC & AUGACA \\
\hline 4 & GACG & UGACA & ACGACA \\
\hline 5 & ACGA & GACAA & CAUGAC \\
\hline
\end{tabular}

\section{g}
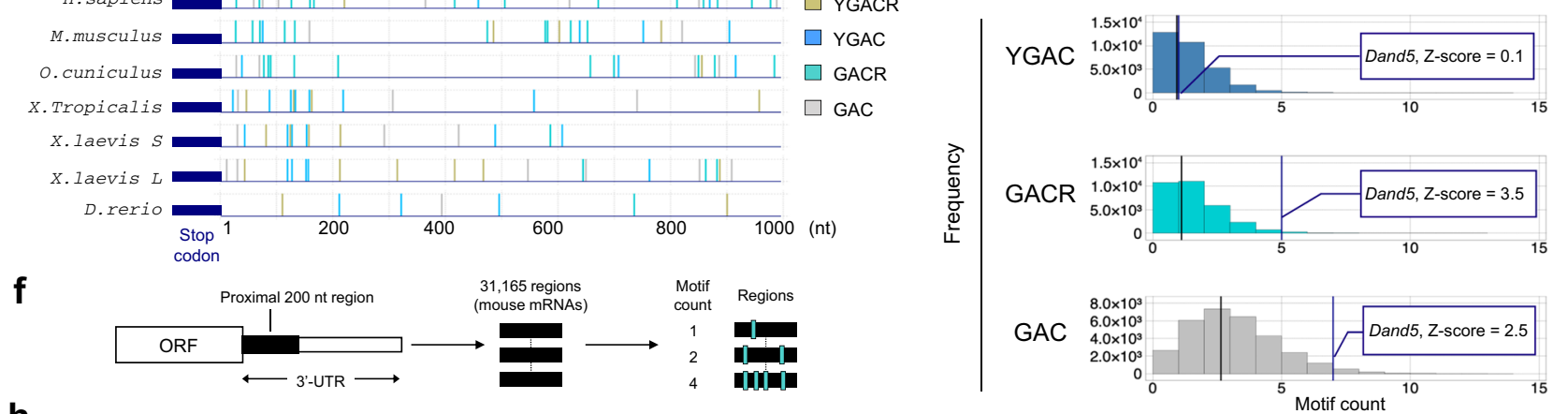

h

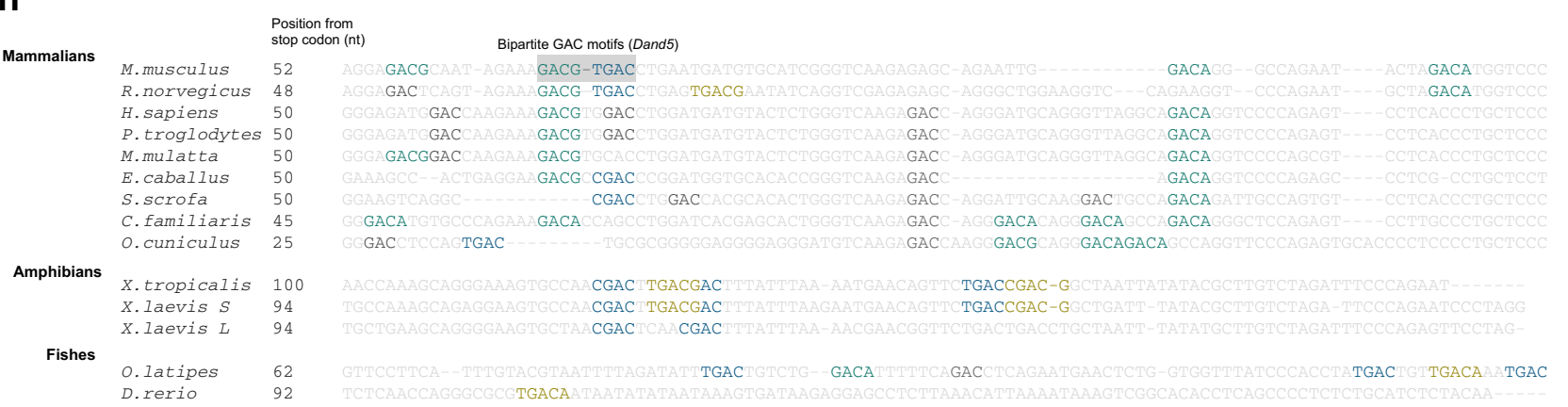

Fig. 6 Screen for Bicc1-binding motifs in RNA. a Schematic representation of RNA Bind-n-Seq (RBNS), which determines RNA motifs enriched by target proteins with the use of a random RNA sequence library. 293FT cells were transfected with a plasmid for overexpression (O/E) of FLAG-tagged Bicc1. Cell lysates containing the Bicc1-FLAG protein were then mixed with a random RNA sequence library, and resulting RNA-protein complexes were isolated by immunoprecipitation with magnetic bead-conjugated antibodies to FLAG. Finally, the isolated RNA sequences were converted to a DNA library by RT-PCR for deep sequencing. $\mathbf{b}$ Analysis of the RBNS data set. The number of each k-mer (where $k=4,5$, or 6 ) RNA sequence was compared between cells transfected with the Bicc1-FLAG expression plasmid and those subjected to mock transfection (control). c Motif logos generated from aligned hexamers that were enriched by Bicc1-FLAG. d 4-mer, 5-mer, and 6-mer sequences ranked by their relative frequencies in Bicc1-FLAG versus control RBNS data. e Maps of GAC-containing motifs in the $3^{\prime}$-UTR of Dand5 mRNAs for the indicated species. $\mathbf{f}$ Schematic representation of metagene analysis for the 200 nucleotide proximal region of the 3'-UTR of mouse mRNAs. A total of 31,165 regions extracted from mouse genes (mm10) was searched with the indicated target motifs. $\mathbf{g}$ Histogram of motif frequency revealed by metagene analysis. The vertical lines indicate the averaged frequency of each target motif (black) and the frequency of each target motif in the 200-nucleotide proximal region of the 3'-UTR of Dand5 mRNA (blue), respectively. $\mathbf{h}$ Multiple sequence alignment of a conserved segment within the proximal 200 nucleotides of mammalian, amphibian, and fish Dand5 3'-UTRs. Colors highlight GAC motifs.

degradation of the Dand5 mRNA in response to Pkd2 stimulation by leftward fluid flow.

Biccl binds to various target mRNAs, but no consensus motif recognized by this protein has been identified. Our comparison of various mammalian Dand5 genes revealed that conservation among the $3^{\prime}$-UTR sequences is largely limited to the 200nucleotide proximal-most region which we found to be required for the establishment of L-R asymmetry of Dand5 mRNA in crown cells. The only conserved features in this region or elsewhere in the 3'-UTR among mammalian Dand5 genes consist of a predicted stem-loop with a GACGUGAC motif at its base, and enrichment of additional GAC motifs. Using EMSA and coimmunoprecipitation assays, we found that the GACGUGAC sequence mediates specific binding to Biccl. Since KH domains 
a

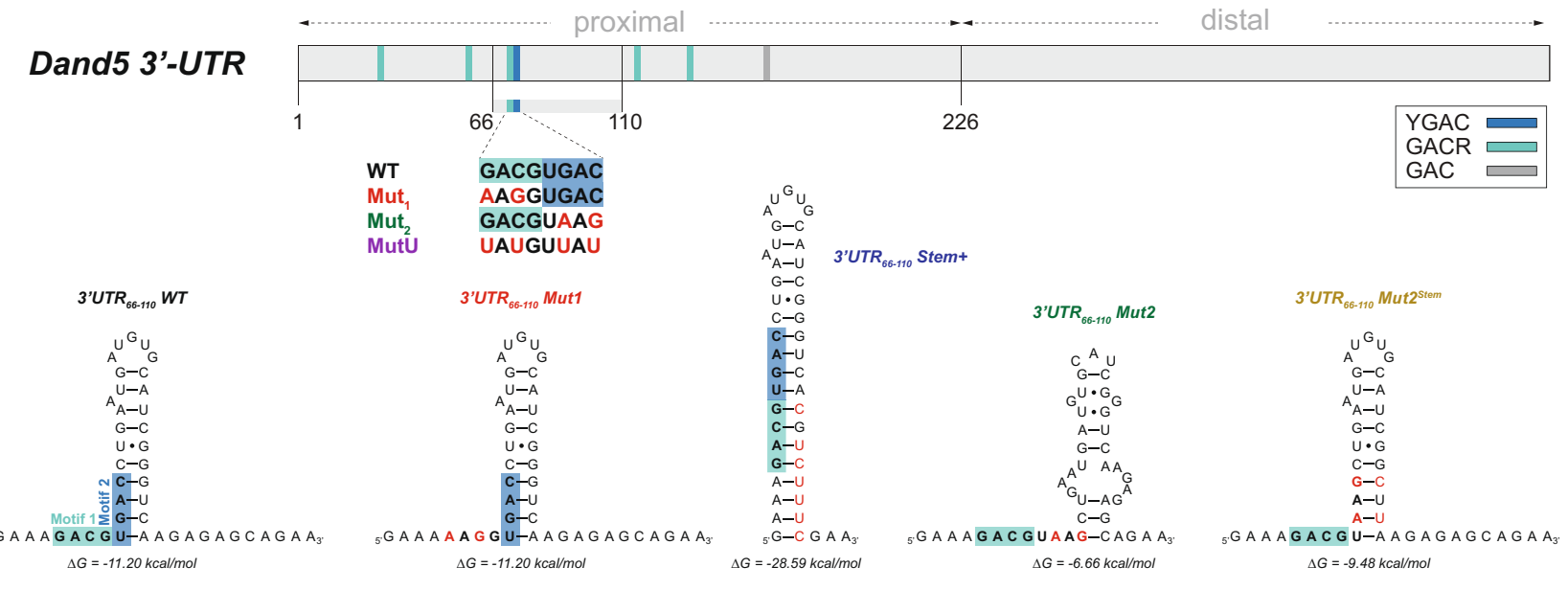

b
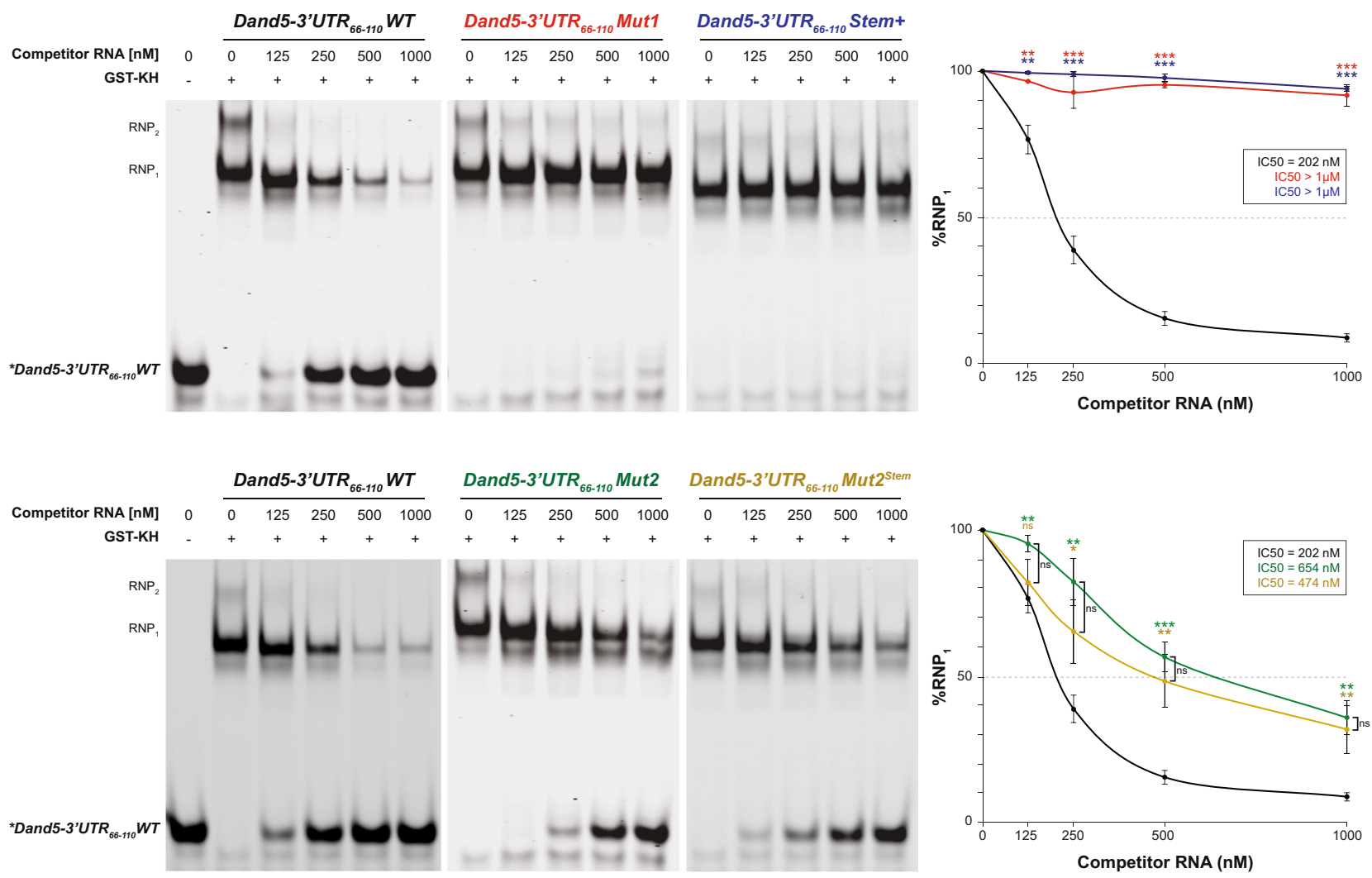

C

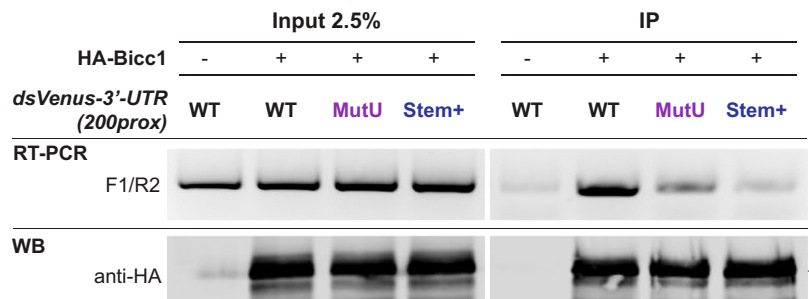

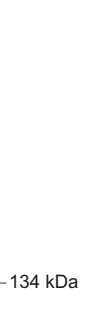

accommodate single-stranded 4-nucleotide sequences $^{23}$, and since our RBNS analysis in vitro revealed a preference of Biccl for GACR and YGAC motifs, Biccl binding to GACGUGAC in the Dand5 3'-UTR where these motifs are juxtaposed likely involves at least two $\mathrm{KH}$ domains. While GACG was essential for specific Biccl binding in vitro, UGAC further increased this interaction. Conversely, we found that a mutation in the $\mathrm{KH} 2$ domain blocked binding to the Dand5 3'-UTR in HEK293T cells, in line with recent findings on Cripto1 mRNA binding in Xenopus ${ }^{32}$. Since maximal binding to the Dand5 $3^{\prime}$-UTR also required the KH1 domain, KH2 and the GACG motif in Dand5 mRNA may be essential to prime a complex that is then stabilized by $\mathrm{KH} 1$ binding to the adjacent UGAC sequence. In line with this model, at least the GACG motif should be accessible, whereas the UGAC 
Fig. 7 A conserved GACGUGAC motif recruits Bicc1 to the proximal Dand5 3'-UTR. a In vitro-transcribed Dand5 3'-UTR66-110 RNA and mutant versions used as competitors in EMSA assays to assess the specificity of Bicc1 binding. Their secondary structures and minimum free energies ( $\Delta \mathrm{G}$ ) were predicted by the RNAfold server at http://rna.tbi.univie.ac.at/cgi-bin/RNAWebSuite/RNAfold.cgi. b EMSA analysis of competitive binding of Bicc1 KH domains to in vitro transcripts of WT or mutant Dand5 3'-UTR $66-110$ against the fluorescent wild-type Dand5 3'-UTR $66-110$ RNA probe. Increasing amounts of unlabeled competitors were added to pre-assembled complexes of fluorescent probe with recombinant GST-KH fusion protein. The \% of residual RNP relative to the indicated competitor RNA concentration, and the half maximal inhibitory concentrations (IC50) of each competitor are indicated in the graphs to the right. $P$ values (asterisks) above the curves refer to differences between each mutant versus the WT RNA, whereas differences between mutants ( $n$.s.: not significant) are marked by brackets. c RT-PCR analysis of WT and mutant dsVenus-Dand5 3'-UTR(200prox) mRNAs in anti-HA immunoprecipitates of HABicc1 from cytoplasmic extracts of HEK293T cells expressing the indicated constructs. Control anti-HA Western blots (WB) of inputs (2.5\%) and immunoprecipitates (IP) are shown below, and RT-qPCR analysis of immunoprecipitates to the right. Enrichment of co-immunoprecipitated mRNA relative to the input is shown as the percentage of enrichment of WT mRNA (100\%). All quantifications represent means \pm SD from three independent experiments. ${ }^{\star} p<0.05,{ }^{\star \star} p<0.01,{ }^{\star \star \star} p<0.001$ (two-sided Student's $t$-test). Source data are provided as a Source Data file.

motif resides in the stem of a predicted stem-loop (Fig. 7a). Moreover, in the Biccl-related C. elegans protein GLD-3, the $\mathrm{KH} 1$ domain is structurally independent, whereas the other $\mathrm{KH}$ domains are interconnected through extended $\beta$-sheets to form a rigid platform ${ }^{33}$. Thus, the $\mathrm{KH} 1$ domain may be flexible enough to pry open the UGAC motif within the stem once the necessary entropy cost is diminished by prior binding of GACG to $\mathrm{KH} 2$ (Supplementary Fig. 9). By a related mechanism, one continuous surface of two $\mathrm{KH}$ domains in the bacterial protein NusA can melt a stem-loop in associated RNA to accommodate an extended sequence of 11 nucleotides ${ }^{34}$. The apparent conservation of the consensus sequence $\mathrm{GACN}_{1-2} \mathrm{GAC}$ in mammalian Dand5 3 '-UTRs suggests that Bicc1 may recognize this extended motif, with a possible contribution of the proximal poly-purine tract $\mathrm{R}_{3-4}$ and a central linker $\mathrm{N}_{1-2}$ of variable length that could be adapted to species-specific constraints in Biccl. Indeed, similar extended motifs of two GAC sequences preceded by a poly-purine tract are conserved among mammals and in Xenopus (Fig. 6h), where they are part of a 36-nucleotide region that is required for flow-induced decay of the Xenopus Dand5S mRNA (Maerker et al., accompanying paper) ${ }^{21}$. However, these findings do not exclude the possibility that Bicc1 may diversify its panel of target transcripts by using different combinations of its three $\mathrm{KH}$ domains to bind alternative combinations of GAC motifs.

Biccl binds to various target mRNAs. Biccl is expressed not only in peri-nodal cells but also in pit cells with motile cilia (Fig. 4). In about a half of Bicc1 ${ }^{\Delta \operatorname{ex} 1 / \Delta \operatorname{ex} 1}$ embryos, motile cilia at the node exhibited polarity defects (Supplementary Fig. 4), suggesting that Biccl has additional target mRNAs in pit cells. On other target mRNAs such as mouse Adcy6 or Xenopus Cripto1, Bicc1 has been shown to inhibit their translation ${ }^{11,27,35}$. In crown cells of the mouse node as well as in ciliated cells at the gastrocoel roof plate of Xenopus embryos, however, the level of Dand5 mRNA eventually becomes asymmetric $(\mathrm{R}>\mathrm{L})$ in response to nodal flow $^{9}$, suggestive of increased mRNA decay. Bicc1mediated regulation of certain mRNAs has been shown to involve microRNAs (miRNAs) ${ }^{11,36}$, and miRNA-mediated inhibition of target mRNAs typically involves both translational repression and mRNA decay ${ }^{37}$ mediated by Ccr4-Not ${ }^{38}$. Consistent with a potential role for miRNAs in the regulation of Dand5 mRNA in crown cells of the mouse embryo, deletion of Dicer in these cells abolished asymmetric Nodal expression in the lateral plate (Maerker et al., accompanying paper) ${ }^{21}$. However, given that no miRNA seed sequences are highly conserved in the $3^{\prime}$-UTR of mammalian Dand5 mRNAs (Fig. 3a) and that Ccr4Not can also be recruited by specific RNA-binding proteins independently of miRNAs ${ }^{39}$, the regulation of Dand 5 mRNA by Biccl may be mediated by an miRNA-independent pathway. In all, our data indicate that inhibition of Dand5 expression at the mouse node is dependent on the $3^{\prime}$-UTR of the mRNA and mediated by Bicc1 and Cnot3 at the level of mRNA decay.

The degradation of Dand5 mRNA in crown cells occurs predominantly on the left side of the mouse node. How can the Bicc1-Cnot3 pathway be activated on the left side but not on the right side? Several lines of evidence implicate $\mathrm{Ca}^{2+}$ in the decay of Dand5 mRNA. First, the Dand5-dependent asymmetry $(\mathrm{R}<$ L) of Nodal activity in crown cells is disrupted by various $\mathrm{Ca}^{2+}$ blockers including thapsigargin ${ }^{5}$ and ionophore ${ }^{40}$. Furthermore, $\mathrm{R}>\mathrm{L}$ asymmetric expression of the NDE-Hsp-dsVenu'-3'-UTR transgene at the node in the present study was impaired by thapsigargin treatment and by the absence of $\mathrm{Pkd} 2$. The decay of Dand5 mRNA may therefore be induced by the $\mathrm{Ca}^{2+}$ influx triggered by sensing of nodal flow by the immotile cilia of crown cells ${ }^{6}$. The resulting increase in the cytosolic concentration of $\mathrm{Ca}^{2+}$ may influence, directly or indirectly, either the interaction with a component (X in Fig. 9) of the pathway responsible for mRNA decay (such as between Biccl and Dand5 mRNA, or between Biccl and Ccr4-Not) or the deadenylase activity of Ccr4-Not. Of note in this regard, crown cells at the mouse node express calmodulin and proteins such as Inversin with a calmodulin-binding motif, some of which are known to be essential for L-R asymmetry ${ }^{41,42}$. Future studies are thus warranted to probe further the role of $\mathrm{Ca}^{2+}$ in the regulation of Dand5 mRNA decay.

\section{Methods}

Mouse strains. All mouse experiments were performed in accordance with guidelines of the RIKEN Center for Biosystems Dynamics Research and under an institutional license (A2016-01-6). Mice were maintained in the animal facility of the RIKEN Center for Biosystems Dynamics Research. Noto-Cre ${ }^{\text {ERT2 }}$ mice $^{30}$, Cnot $3^{\text {flox } /+}$ mice ${ }^{29}$, iv mice ${ }^{19}$, and $P k d 2$ mutant mice ${ }^{3}$ were described previously. Expression of the Noto-Cre $e^{\text {ERT2 }}$ transgene in embryos was induced by oral administration of tamoxifen (Sigma) in corn oil to pregnant mice at a dose of $5 \mathrm{mg}$ both 24 and $12 \mathrm{~h}$ before the late headfold stage.

Cell lines. HEK293T and HeLa cells were cultured in DMEM (Sigma) supplemented with 10\% FBS (Sigma), 1\% GlutaMAX (Thermo Fisher Scientific), and 1\% gentamicin (Thermo Fisher Scientific). The cells were transfected with expression plasmids with the use of jetPEI (Polyplus Transfection). IMCD cells were cultured in DMED/F12 (1:1) (Gibco 11320-033) with 10\% FBS. For RBNS analysis, the human embryonic kidney cell line 293FT (Invitrogen, \#R70007) was cultured under a humidified atmosphere of $5 \% \mathrm{CO}_{2}$ at $37^{\circ} \mathrm{C}$ in Dulbecco's modified Eagle's medium (DMEM) (Nacalai tesque, \#08459-64) supplemented with $10 \%$ fetal bovine serum (FBS) (Biosera, \#554-02155, lot \#10259), 2 mM L-glutamine (Thermo Fisher Scientific, \#25030-081), $1 \times$ MEM Nonessential Amino Acids (Thermo Fisher Scientific, \#11140-050), and $1 \mathrm{mM}$ sodium pyruvate (Sigma, \#S8636-100ML).

Transgene design. To generate the transgene NDE-Hsp-dsVenus- $3^{\prime}-U T R$ that confers expression of dsVenus at the node of mouse embryos, the dsVenus coding sequence was linked to the $1.2 \mathrm{~kb}$ DNA sequence for the $3^{\prime}$-UTR of mouse Dand5 mRNA and placed under the control of four copies of the crown cell-specific enhancer (NDE) of the mouse Nodal gene and the mouse Hsp68 promoter. 
a
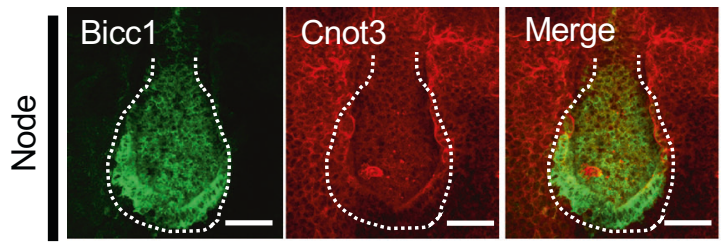

Super resolution
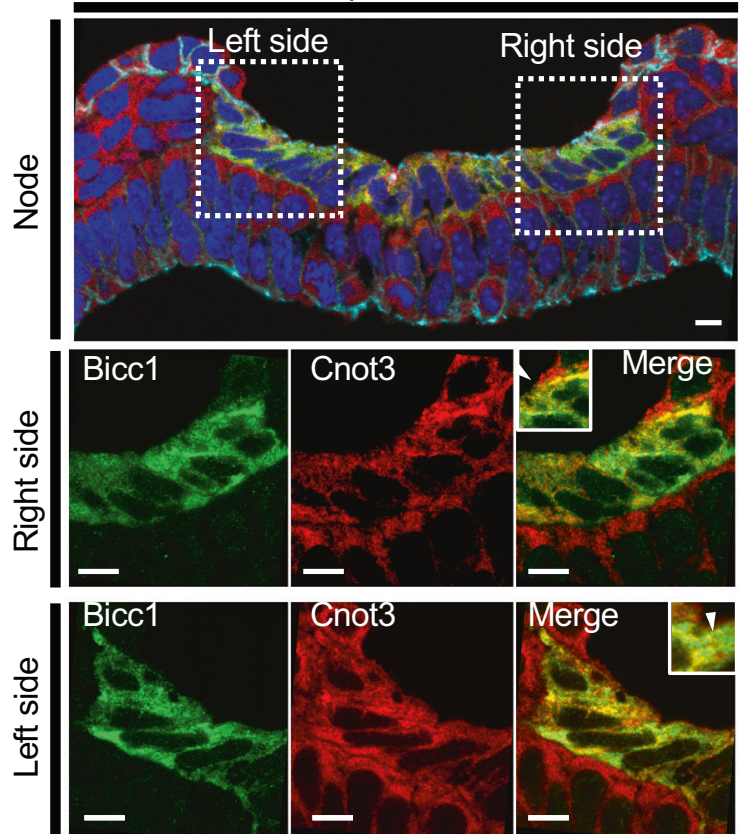

b

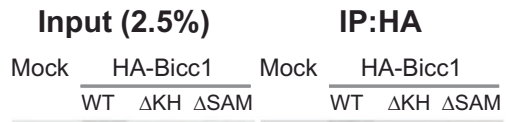

HA-Bicc1
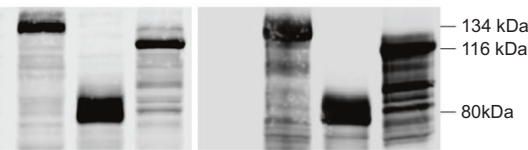

Cnot1 $=\Longleftarrow=-266 \mathrm{kDa}$
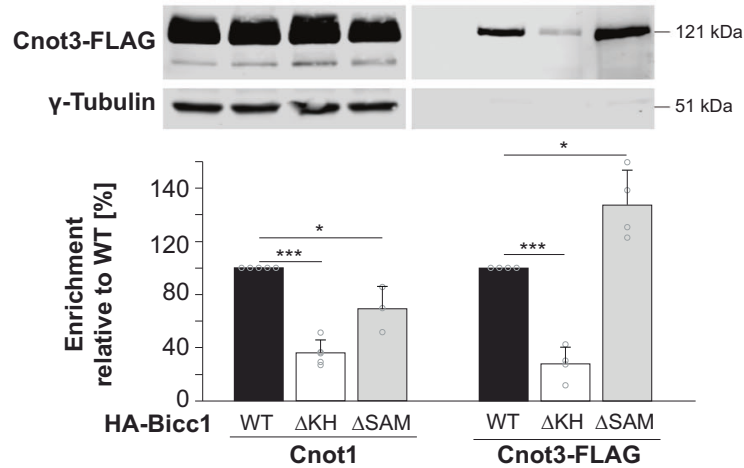

C

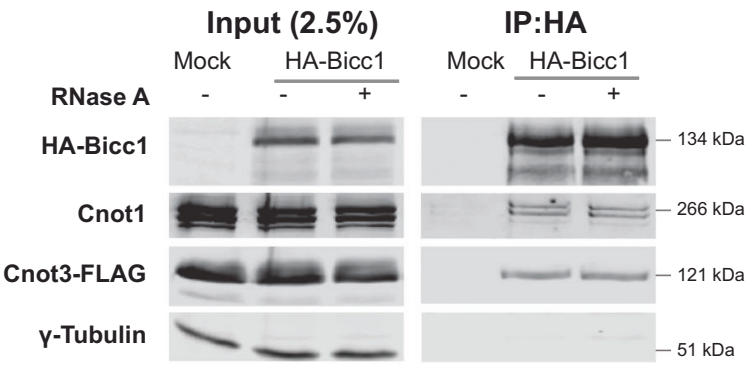

d

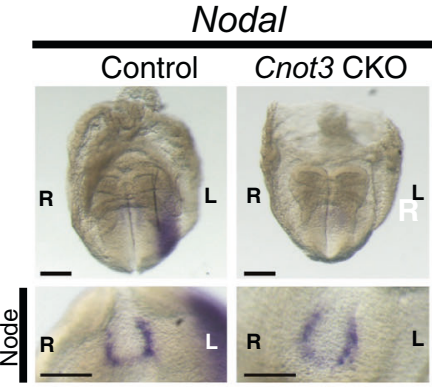

f

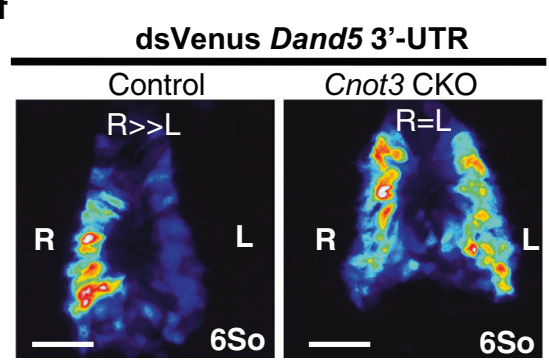

e

Dand5

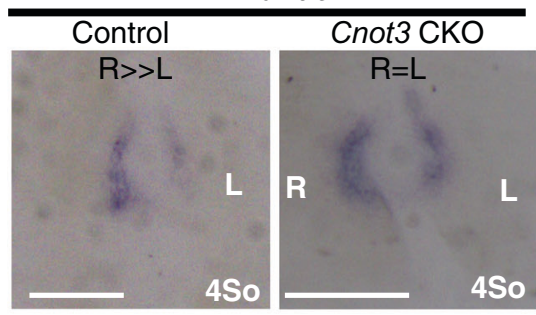

g

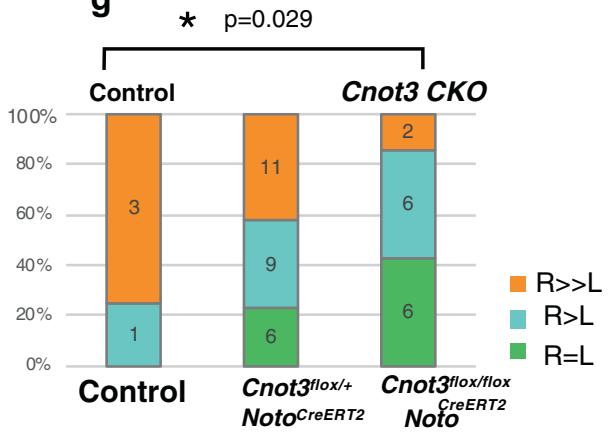

Transgenes containing only the proximal 200 bp region (200prox) or a truncated $3^{\prime}$-UTR lacking the proximal or distal 200 bp region (NDE-Hsp-dsVenus- $3^{\prime}$ UTR $\Delta 200$ prox or $\Delta 200$ dist) were generated analogously.

Generation of transgenic and mutant mice. Dand $5^{\Delta 200 p r o x / \Delta 200 p r o x}$ embryos (Fig. S1) and Bicc1 ${ }^{\Delta \operatorname{ex} 1 / \Delta \text { ex1 }}$ embryos (Fig. S2) were generated with the use of the
CRISPR/Cas9 system. For the generation of transgenic mice, each transgene was microinjected into the pronucleus of fertilized eggs obtained by crossing C57BL/6 J female and male mice. The eggs were then transferred to pseudopregnant mothers. In some instances (embryos shown in Fig. 3B, D, E), transgenic embryos were directly examined before the establishment of stable transgenic lines. Dand5 and Bicc1 mutant mice and embryos were genotyped by genomic PCR analysis with the primers indicated in Supplementary Figs. 3 and 4. 
Fig. 8 Decay of Dand5 mRNA on the left side of the node requires the Ccr4-Not deadenylase complex. a Immunofluorescence staining of Bicc1 and Cnot3 in node cells of the WT mouse embryo at E8.0 shown as whole mounts (top row) and in a transverse section (second row). Nuclei were stained with 4',6-diamidino-2-phenylindole (DAPI). Regions of the node indicated by the dotted rectangles are shown in bottom rows at higher magnification. Note that Bicc1 and Cnot3 proteins are co-localized in the cytoplasm (arrowheads). Scale bars in the top panels, $50 \mu \mathrm{m}$. Scale bars in the super resolution panels, $5 \mu \mathrm{m}$. Images shown are representative of at least two independent experiments. b Extracts of HEK293T cells transfected with expression plasmids for Cnot3-FLAG and either WT or the indicated mutant forms of HA-Biccl (or with the corresponding empty vector, Mock) were subjected to immunoprecipitation with antibodies to HA. The resulting immunoprecipitates as well as a portion (2.5\%) of the original cell extracts were then subjected to immunoblot analysis with antibodies against HA, Cnot1, FLAG, or $\gamma$-tubulin (loading control). The enrichment of Cnot subunits relative to the indicated HA-Bicc1 proteins in the IP fractions is shown as a percentage of the value for HA-Bicc1 WT. Data are means \pm SD from at least three independent experiments. The precise value of replicate numbers is indicated by grey circles. For the anti-Cnot 1 Western blot, $n=5$ for $\Delta K H, n=3$ for $\Delta S A M$ and $n=5$ for wild-type. For anti-FLAG Western blots, $n=4$ for $\Delta \mathrm{KH}, \Delta$ SAM and wild-type. ${ }^{\star} p<0.05,{ }^{\star \star} p<0.01,{ }^{\star \star \star} p<0.001$ (two-sided Student's $t$-test). Source data are provided as a Source Data file. c Co-immunoprecipitation of Cnot1 and Cnot3-FLAG with HA-Bicc1 before and after RNase A treatment of cell extracts was performed as in (b). d WISH analysis of Nodal expression in control and Cnot3 CKO embryos at E8.0. The node region of the same embryo is shown below. Note that Nodal expression at the node was maintained at the node but that in LPM was lost in the mutant embryo. Scale bars, 100 um. e WISH analysis of Dand5 mRNA in control and Cnot3 CKO embryos at four- to six-somite stages. The R $>\mathrm{L}$ asymmetry of Dand5 mRNA in the control embryo is attenuated or lost in the Cnot3 CKO embryos. Scale bars, $100 \mu \mathrm{m}$. f Fluorescence of ds Venus in control and Cnot3 CKO embryos harboring the NDE-Hsp-dsVenus-3'-UTR transgene at the four-somite stage. Note that Venus fluorescence is increased on the left side of the Cnot3 CKO embryo. Scale bars, $50 \mu \mathrm{m}$. Images shown in (d), (e), and (f) are representative of thirteen, fourteen, and seven embryos, respectively. $\mathbf{g}$ Summary of the L-R patterns of Dand5 mRNA expression. The $p$ value of the difference between the indicated genotypes was determined using the two-sided Wilcoxon rank sum test. The numbers in bars indicate the number of embryos per genotype.

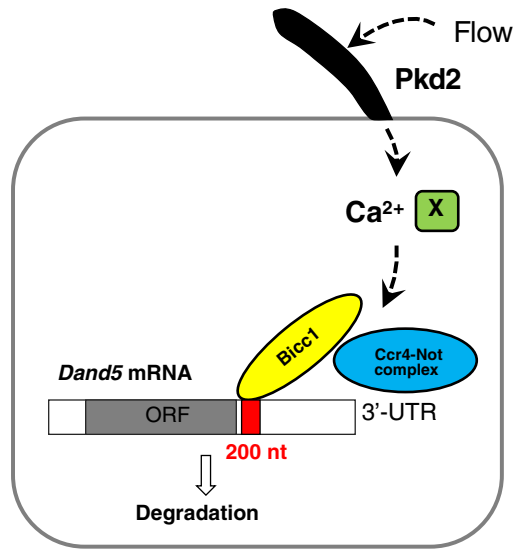

Fig. 9 Model for the degradation of Dand5 mRNA in crown cells of the node in response to nodal flow. See text for details.

Plasmids, cloning, and mutagenesis. The plasmid pCMV6-Entry Cnot3-MycFLAG encoding mouse Cnot3 was obtained from Origene (MR210463). The pCMV-SPORT6 HA-Biccl plasmids for WT, $\triangle \mathrm{KH}, \triangle \mathrm{SAM}$, and mutD forms of mouse Biccl were previously described ${ }^{10,12}$. Vectors encoding mutKH1, mutKH2, mutKH3, or mutKH1,2,3 were generated by overlap extension PCR and subcloned between the SalI and BstBI sites of pCMV-SPORT6 HA-Biccl. Vectors encoding the Dand5 $3^{\prime}$-UTR variants mutU and Stem + were generated by overlap extension PCR and subcloned at the unique NotI site of pEFBOS Dand5-3'UTR(200prox). The sequences of all mutated constructs were verified by Sanger sequencing.

Recombinant protein production. Recombinant GST-Biccl-KH was were expressed in E. coli BL21(DE3)pLysS cells (Promega) and purified by affinity chromatography on glutathione-sepharose beads (GE Healthcare) in $50 \mathrm{mM}$ Tris$\mathrm{HCl}$ ( $\mathrm{pH}$ 7.5), $200 \mathrm{mM} \mathrm{NaCl}, 1 \mathrm{mM}$ DTT. After elution in $50 \mathrm{mM}$ Tris- $\mathrm{HCl}(\mathrm{pH} 8)$, $150 \mathrm{mM} \mathrm{NaCl}, 10 \mathrm{mM}$ reduced glutathione, $1 \mathrm{mM}$ DTT, the protein was dialyzed and concentrated on Amicon Ultra-0.5 mL column (Merck, UFC500396).

WISH analysis. WISH was performed according to standard procedures with digoxigenin-labeled riboprobes specific for Dand5, Nodal, and dsVenus mRNAs ${ }^{43}$.

Immunofluorescence analysis. Dissected embryos were fixed with $4 \%$ paraformaldehyde, dehydrated with methanol, and permeabilized with phosphatebuffered saline (PBS) containing $0.1 \%$ Triton X-100. They were incubated overnight at $4{ }^{\circ} \mathrm{C}$ with primary antibodies at the following dilutions: anti-Bicc1 $(1: 100$ dilution, rabbit polyclonal, Sigma), anti-acetylated tubulin (1:100 dilution, mouse monoclonal, Sigma), anti-ZO1 (1:10 dilution, mouse monoclonal, clone ZO1-1A12, Invitrogen), anti-Cnot3 (mouse monoclonal, 1:50-100 dilution, described previously $\left.{ }^{16}\right)$ and anti-Odf2 (1:100 dilution, rabbit polyclonal, Abcam). The embryos were washed with PBS containing $0.1 \%$ Triton X-100 before exposure to Alexa Fluor-conjugated secondary antibodies (Invitrogen) and DAPI (Wako). The node of stained embryos was excised, placed on a slide glass with silicone rubber spacers, covered with a cover glass, and imaged with an Olympus FV1000 or FV3000 confocal microscope. To localize Biccl and Cnot3 in node cells, cryosections were prepared from immunostained node samples and imaged by superresolution AiryScan mode using an LSM880 confocal microscope (Zeiss). For immunostaining of HeLa cells, cells cultured in six-well plates and transfected with $1 \mu \mathrm{g}$ of each expression vector for $24 \mathrm{~h}$ were transferred to sterile coverslips in the wells of a 24-well plate. After culture for an additional $24 \mathrm{~h}$, the cells were fixed for $10 \mathrm{~min}$ at $-20^{\circ} \mathrm{C}$ in methanol, washed with PBS, and incubated at room temperature first for $1 \mathrm{~h}$ in PBS containing $1 \%$ bovine serum albumin as a blocking agent and then for $2 \mathrm{~h}$ in the blocking solution containing antibodies to HA (1:500, rabbit monoclonal, Sigma) and to FLAG (1:500, mouse monoclonal clone M2, Sigma). After washing with PBS, the cells were incubated for $1 \mathrm{~h}$ at room temperature with Alexa Fluor 568- or Alexa Fluor 647-conjugated secondary antibodies to mouse or rabbit immunoglobulin G, respectively, in blocking buffer containing DAPI (1:10,000 dilution). Images were acquired with a Zeiss LSM700 confocal microscope and deconvoluted using Huygens Remote Manager (https:// hrm-biop.epfl.ch/).

Observation of nodal flow by PIV analysis. Nodal flow was observed by multipoint scanning confocal microscopy. Particle image velocimetry (PIV) analysis was performed as described previously ${ }^{18}$. Recovered embryos were first cultured under $5 \% \mathrm{CO}_{2}$ for $30 \mathrm{~min}$ at $37^{\circ} \mathrm{C}$ in DMEM supplemented with $75 \%$ rat serum The region containing the node was then excised, and the node cavity was filled with DMEM supplemented with 10\% FBS and $0.2 \mu$ m-diameter fluorescent microbeads (Invitrogen, F8811). The motion of the beads was monitored for $10 \mathrm{~s}$ in planes of +5 and $+10 \mu \mathrm{m}$ relative to the cavity ( 21 frames per second) with the use of a CSU-W1 confocal unit (Yokogawa) and an iXon-Ultra EMCCD camera (Andor Technology) connected to an IX83 microscope (Olympus) fitted with a $\times 60$ objective lens. Time-series images for PIV analysis were captured at a resolution of 512 by 512 pixels and were processed with interrogation windows of 16 by 16 pixels with $50 \%$ overlap, corresponding to a spatial resolution of 4.3 by $4.3 \mathrm{~mm}$. The timeaveraged velocity distributions were calculated for 10 -s intervals.

Quantitative analysis of basal body position. The average basal body position (ABP) representing the relative position of the basal body in each node cell along the A-P axis (vertical) was analyzed as described previously ${ }^{44,45}$. Briefly, confocal images of the node stained with antibodies to Odf2 and to ZO-1 were obtained to determine the position of the basal body in each node cell. For characterization of the shape and orientation of the node cells, the outline of each cell was calculated from the pattern of ZO-1 staining with the use of an ImageJ software plugin (http:// rsb.info.nih.gov/nih-image) to apply watershed segmentation. The basal body was traced manually according to the Odf2 staining image, and the $x$ and $y$ coordinates of each basal body were recorded with the use of the graphical user interface (GUI) of MATLAB software. The relative value for the position of the basal body in each cell was calculated from the coordinate data with the anterior and posterior ends of the cell expressed as -1.0 and +1.0 , respectively.

Mouse embryo culture. Embryos were cultured under conditions of artificial fluid flow as described previously ${ }^{20}$. Briefly, embryos were collected at E7.5. Those at the early headfold stage were selected and cultured for $1 \mathrm{~h}$ with rotation under $5 \% \mathrm{CO}_{2}$ 
at $37^{\circ} \mathrm{C}$ in DMEM supplemented with $75 \%$ rat serum. They were transferred to a flow chamber, were cultured in DMEM with $50 \%$ rat serum for $7 \mathrm{~h}$, and were then subjected to conventional rotation culture for additional $\sim 2 \mathrm{~h}$. Where indicated, thapsigargin was added to the medium at a final concentration of $500 \mathrm{nM}$ for $1 \mathrm{~h}$ (during the initial rotation culture) and the embryos were then cultured in the absence of the drug. The node cavity was imaged with an Olympus FV1000 confocal microscope.

Imaging and analysis of dsVenus. The node of NDE4-hsp-dsVenus-Dand5-3'UTR transgenic embryos was excised in DMEM supplemented with $75 \%$ rat serum, placed on glass slides with silicone rubber spacers and glass cover slips for imaging with an Olympus FV1000 confocal microscope. The sum of XYZ images was obtained with Fiji/ImageJ as a 32-bit image. ROIs were set so as to include all crown cells on the left or right side of the node, and cells with fluorescence were selected. The mean signal intensity was measured for the left and right sides of the node, and the R/L ratio of the mean intensity was then determined.

Protein co-immunoprecipitation and Western blot analysis. HEK293T cells in two $10-\mathrm{cm}$ dishes per condition $\left(10^{7}\right.$ cells per dish) were transfected with expression plasmids for Cnot3-FLAG $(2 \mu \mathrm{g})$ and either HA-Biccl(WT) $(2 \mu \mathrm{g})$, HA$\operatorname{Biccl}(\Delta \mathrm{KH})(1 \mu \mathrm{g})$, HA-Biccl( $\Delta \mathrm{SAM})(4 \mu \mathrm{g})$, or HA-Biccl(mutD) $(4 \mu \mathrm{g})$. After incubation for 3 days, cells from the two dishes for each condition were then washed with PBS and pooled in extraction buffer containing $20 \mathrm{mM}$ Tris- $\mathrm{HCl}$ ( $\mathrm{pH}$ 7.4), $2.5 \mathrm{mM} \mathrm{MgCl}_{2}, 100 \mathrm{mM} \mathrm{NaCl}, 5 \%$ glycerol, $1 \mathrm{mM}$ dithiothreitol (DTT), $0.05 \%$ Nonidet P-40 (NP-40), RNasin (Promega), phosphatase inhibitors (Sigma), and protease inhibitors (Roche). Total cell extracts were prepared by ultrasonication followed by two rounds of centrifugation at $10,000 \times g$ for $5 \mathrm{~min}$ at $4{ }^{\circ} \mathrm{C}$ to remove debris. Supernatants were incubated with $20 \mu \mathrm{l}$ of anti-HA beads (Sigma) for $2.5 \mathrm{~h}$ at $4{ }^{\circ} \mathrm{C}$ on a rotating wheel. For RNase treatment, cell extracts were supplemented with $12.5 \mu \mathrm{g} / \mathrm{mL}$ of RNase A (Roche) and incubated for $10 \mathrm{~min}$ at RT prior to incubation on beads. The beads were rinsed four times for $10 \mathrm{~min}$ in wash buffer (20 mM Tris- $\mathrm{HCl} \mathrm{pH}$ 7.4, $2 \mathrm{mM} \mathrm{MgCl} 2,200 \mathrm{mM} \mathrm{NaCl}, 1 \mathrm{mM} \mathrm{DTT}$, and $0.1 \% \mathrm{NP}$ 40), suspended in Laemmli buffer, and directly loaded on reducing SDS PAGE gels to size-separate eluted proteins. For immunoblot analysis, proteins were transferred to nitrocellulose membranes, blocked with skim milk, and incubated overnight with the antibodies indicated, and with fluorescently labeled secondary antibodies for analysis on a Odyssey CLx scanner (LI-COR Biosciences). The signal of coimmunoprecipitated protein was normalized relative to the signal of Biccl in the IP fraction.

RNA co-immunoprecipitation in HEK293T cells. HEK293T cells were transfected as described above, but with an expression plasmid for the ORF of dsVenus linked to the WT or $\Delta 200$ forms of the $3^{\prime}$-UTR sequence of mouse Dand5 mRNA ( $2 \mu \mathrm{g}$ per dish) together with a plasmid encoding WT or $\triangle \mathrm{KH}$ mutant forms of HABiccl (2 or $1 \mu \mathrm{g}$ per dish, respectively). Cytoplasmic extracts prepared after $36 \mathrm{~h}$ as described above were passaged eight times through a syringe needle (no. 30) followed by centrifugation twice at $10,000 \times g$ for $5 \mathrm{~min}$ at $4{ }^{\circ} \mathrm{C}$. After setting aside $5 \%$ of each supernatant as input, the remainder was immunoprecipitated as described above. While a portion of the beads (10\%) was analyzed by immunoblotting as described above, the remainder of the beads and half of the input samples were subjected to phenol-chloroform extraction. After ethanol precipitation isolated RNA was treated with RQ1 DNase (Promega) and converted to cDNA by PrimeScript Reverse Transcriptase Kit (Takara). The resulting cDNA was then subjected to PCR or qPCR using Phire Green Hot Start II PCR Master Mix (Promega) or GoTaq qPCR Master Mix (Promega), respectively, with the following primers: F1 (GCTGAGCATCCTAGAGGAATGC), F2 (TGCCA-

CAATCACTAACTCACGTC, R1 (TGTCTTGGACACTGGGACGC) and R2 (TAAACCCATGACTGGGGGACCATGTCTAG) for the 3'-UTR of Dand5 mRNA, and ACAGAGCCTCGCCTTTGCC and CTCCATGCCCAGGAAGGAAGG (forward and reverse, respectively) for $\beta$-actin mRNA. The amount of coimmunoprecipitated mRNA as a percentage of the input was calculated with the formula: $100 \times 2\left[\left(\mathrm{Ct}(\mathrm{Input})-\log _{2}(100 / 2.5)-\mathrm{Ct}(\mathrm{IP})\right]\right.$, where the value 2.5 represents $2.5 \%$ of the original cytoplasmic extract, and where $\mathrm{Ct}$ is the cycle threshold for qPCRs on input or immunoprecipitate (IP) samples. Fold enrichment was calculated relative to cells transfected with the corresponding empty vector for HABiccl.

RBNS analysis. RBNS analysis was performed essentially as described by others ${ }^{26}$. A double-stranded DNA template for in vitro transcription of a 20 -mer random RNA library was synthesized with a primer extension reaction in which $100 \mu \mathrm{l}$ of a reaction mixture containing $1 \times$ Platinum SuperFi PCR Master Mix (Thermo Fisher Scientific, \#12358-010), 1× SuperFi GC Enhancer (Thermo Fisher Scientific), $100 \mathrm{nM}$ DNA template oligomer (5'-GAAATTAATACGACTCACTATAGGA

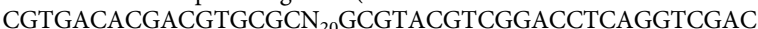
CATGGACGC- $3^{\prime}$, where $\mathrm{N}_{20}$ is the DNA sequence encoding the 20-mer RNA sequence), $100 \mathrm{nM}$ primer (5'-GCGTCCATGGTCGACCTGAGGTCC- $3^{\prime}$ ), and nuclease-free water was incubated at $98{ }^{\circ} \mathrm{C}$ for $130 \mathrm{~s}$, at $50^{\circ} \mathrm{C}$ for $2 \mathrm{~min}$, and then at $72^{\circ} \mathrm{C}$ for $10 \mathrm{~min}$. The synthesized DNA template was purified with the use of a Monarch PCR \& DNA Cleanup Kit (New England Biolabs, \#T1030L). The random
RNA library was then transcribed with the use of a MEGAshortscript T7 Transcription Kit (Thermo Fisher Scientific, \#AM1354) in a reaction mixture containing $1 \times$ Reaction Buffer, $1 \times$ T7 Enzyme Mix, 7.5 mM ATP, 7.5 mM UTP, 7.5 mM GTP, $7.5 \mathrm{mM}$ CTP, and $9.15 \mathrm{pmol}$ of the DNA template. The mixture was incubated at $37^{\circ} \mathrm{C}$ for $6 \mathrm{~h}$, after which the DNA template was digested with TURBO DNase for $30 \mathrm{~min}$ at $37^{\circ} \mathrm{C}$ and the transcribed RNA library ( $5^{\prime}$-GGACGUGACACGACG UGCGCN $_{20}$ GCGUACGUCGGACCUCAGGUCGACCAUGGACGC-3') was purified with an RNA Clean \& Concentrator (Zymo Research, \#R1016).

For generation of an expression vector for Bicc1-FLAG (pcDNA3.1-Bicc1FLAG), the Biccl ORF was first amplified from Biccl/mBS and then inserted between the BamHI and NotI sites of pcDNA3.1/myc-His A (Invitrogen, \#V80020) to generate pcDNA3.1-Bicc1-myc-His A. The sequences corresponding to the $\mathrm{c}$ Myc and His tags in pcDNA3.1-Biccl-myc-His A were replaced with that for the FLAG tag by PCR-based mutagenesis, thereby generating pcDNA3.1-Biccl-FLAG. 293FT cells $\left(3 \times 10^{6}\right.$ cells) were seeded in a 10 - $\mathrm{cm}$ cell culture dish and cultured for $24 \mathrm{~h}$ before transfection for $24 \mathrm{~h}$ with $15 \mu \mathrm{g}$ of pcDNA3.1-Bicc1-FLAG with the use of Lipofectamine 3000 (Thermo Fisher Scientific, \#L3000008) according to the manufacturer's instructions. As a mock transfection control, cells were treated with Lipofectamine 3000 alone. The cells were then collected by centrifugation at $300 \mathrm{xg}$ for $5 \mathrm{~min}$ at $4{ }^{\circ} \mathrm{C}$, washed with ice-cold PBS, and lysed by incubation for $30 \mathrm{~min}$ on ice, with intermittent vortex mixing, in $1 \mathrm{ml}$ of Cell Lysis Buffer (Invitrogen, \#FNN0021) supplemented with $1 \times$ complete Protease Inhibitor Cocktail (Roche, \#04693116001) and $1 \mathrm{mM}$ phenylmethylsulfonyl fluoride (Thermo Fisher Scientific, \#36978). The cell lysates were centrifuged at $15,300 \times \mathrm{g}$ for $10 \mathrm{~min}$ at $4{ }^{\circ} \mathrm{C}$, and the resulting supernatants were collected, assayed for protein concentration with the use of a Pierce BCA Protein Assay Kit (Thermo Fisher Scientific, \#23227), adjusted to a protein concentration of $700 \mu \mathrm{g} / \mathrm{ml}$ with cell lysis buffer, and stored at $-80^{\circ} \mathrm{C}$

The cell lysate containing Bicc1-FLAG was diluted fourfold with the mock cell lysate for immunoprecipitation. For preparation of antibody-coated magnetic beads, $50 \mu \mathrm{l}$ of Dynabeads Protein G (Thermo Fisher Scientific, \#DB10003) were conjugated with $10 \mu \mathrm{g}$ of M2 mouse monoclonal antibodies to FLAG (Sigma, \#F1804) according to the manufacturer's instructions. The antibody-coated beads were suspended in $400 \mu \mathrm{l}$ of Ab Binding \& Washing Buffer [PBS containing 0.02\% Tween-20 (Roche, \#11332465001)] and mixed with $400 \mu \mathrm{l}$ of the cell lysate. After incubation for $1 \mathrm{~h}$ at $4{ }^{\circ} \mathrm{C}$ with rotation, the beads were separated with a magnet and the supernatant removed. The beads were washed with $1 \mathrm{ml}$ of RNP Binding Buffer [20 mM Tris- $\mathrm{HCl}$ (pH 7.4), $2.5 \mathrm{mM} \mathrm{MgCl}, 80 \mathrm{mM} \mathrm{NaCl}, 20 \mathrm{mM} \mathrm{KCl}, 5 \%$ glycerol (Sigma, \#G5516-100ML), 1 mM DTT (Thermo Fisher Scientific, \#A39255), 0.05\% NP-40 (Thermo Fisher Scientific, \#85124)], suspended in $500 \mu \mathrm{l}$ of RNP Binding Buffer containing 500 pmol of the random RNA library, incubated for $16 \mathrm{~h}$ at $4{ }^{\circ} \mathrm{C}$ with rotation, isolated with the magnet, and washed five times with $1 \mathrm{ml}$ of RNP Binding Buffer. The beads were then incubated for $3 \mathrm{~min}$ at $95^{\circ} \mathrm{C}$ in $200 \mu \mathrm{l}$ of an elution buffer (1\% SDS, $10 \mathrm{mM}$ Tris- $\mathrm{HCl}$, and $2 \mathrm{mM} \mathrm{EDTA}$ ) before separation with the magnet, and the supernatant was collected. The eluted RNA was isolated by phenol-chloroform extraction and ethanol precipitation. The RNA pellet was suspended in $24 \mu \mathrm{l}$ of nuclease-free water and subjected to RT with SuperScript IV Reverse Transcriptase (Thermo Fisher Scientific, \#18090010). Prior to reverse transcription, $20 \mu \mathrm{L}$ of purified RNA library was mixed with $1 \mu \mathrm{L}$ of $10 \mu \mathrm{M}$ reverse primer (5'-GCGTCCATGGTCGACCTGAGGTCC-3'), $1 \mu \mathrm{L}$ of $10 \mathrm{mM}$ dNTPs, and $4 \mu \mathrm{L}$ of nuclease water, and then denatured at $65^{\circ} \mathrm{C}$ for $5 \mathrm{~min}$. The mixture was combined with a premix of $8 \mu \mathrm{L}$ of $5 \mathrm{X}$ SSIV Buffer, $2 \mu \mathrm{L}$ of $100 \mathrm{mM}$ DTT, $2 \mu \mathrm{L}$ of RNase OUT (Thermo Fisher Scientific, \#10777019), and $2 \mu \mathrm{L}$ of SuperScript IV Reverse Transcriptase. The mixture was incubated at $50^{\circ} \mathrm{C}$ for $10 \mathrm{~min}$, then at $85^{\circ} \mathrm{C}$ for $10 \mathrm{~min}$. To prepare an input random RNA library, $0.5 \mathrm{pmol}$ of input random RNA library was also reverse transcribed. After digestion of RNA with $2 \mu \mathrm{l}$ of $\mathrm{RNaseH}$ for $20 \mathrm{~min}$ at $37^{\circ} \mathrm{C}$, the cDNA preparation was amplified by PCR in a reaction mixture containing $1 \times$ Platinum SuperFi PCR Master Mix, $5 \mu \mathrm{M}$ forward primer (5'-TCGTCGGCAGCGTCAGATGTGTATAA GAGACAGGGACGTGACACGACGTGC-3'), $5 \mu \mathrm{M}$ reverse primer (5'-GTCT CGTGGGCTCGGAGATGTGTATAAGAGACAGGGCGTCCATGGTCGACCT GAGGTCCGACG- $3^{\prime}$ ), $1 \times$ SuperFi GC Enhancer, and $2 \mu \mathrm{l}$ of $\mathrm{cDNA}$ and adjusted to $25 \mu \mathrm{l}$ with nuclease-free water. The incubation protocol included an initial denaturation at $98^{\circ} \mathrm{C}$ for $30 \mathrm{~s}, 20$ cycles of denaturation at $98^{\circ} \mathrm{C}$ for $10 \mathrm{~s}$ and extension at $72{ }^{\circ} \mathrm{C}$ for $10 \mathrm{~s}$, and a final extension at $72^{\circ} \mathrm{C}$ for $5 \mathrm{~min}$. Single-stranded DNA was digested with Illustra ExoProStar exonuclease I (GE Healthcare Life Sciences, \#US78211), and the PCR products were purified with the use of a MinElute PCR Purification Kit (Qiagen, \#28006) before the addition of Illumina sequencing barcodes by 10 cycles of PCR with index primers from a Nextera XT Index Kit (Illumina, \#FC-131-1001). The reaction mixture contained 1× Platinum SuperFi PCR Master Mix, $5 \mu$ of Index 1 (i7) primer, $5 \mu$ of Index 2 (i5) primer, $1 \times$ SuperFi GC Enhancer, and at most $5 \mu$ of the PCR products, and the volume was adjusted to $50 \mu \mathrm{l}$ with nuclease-free water. The incubation protocol included an initial denaturation at $98^{\circ} \mathrm{C}$ for $30 \mathrm{~s} ; 10$ cycles of denaturation at $98^{\circ} \mathrm{C}$ for $10 \mathrm{~s}$, annealing at $55^{\circ} \mathrm{C}$ for $10 \mathrm{~s}$, and extension at $72^{\circ} \mathrm{C}$ for $20 \mathrm{~s}$; and a final extension at $72^{\circ} \mathrm{C}$ for $5 \mathrm{~min}$. The resulting sequencing library was quantified with the use of a KAPA Library Quantification Kit (Illumina) and Universal qPCR Mix (Kapa Biosystems, \#07960140001). The library was sequenced for 150 cycles on the Illumina MiSeq platform and with a MiSeq Reagent Kit v3 (Illumina, \#MS-1023001). FASTQ files were processed as follows. The adapter sequences of the reads were trimmed by cutadapt 1.10 with parameter $-\mathrm{e}$ set to 0.2 . Low-quality reads 
(averaged quality score of $<30$ ) were filtered with a custom script written in Julia 1.1. The filtered reads were split into overlapping k-mers $(k=4,5$, or 6$)$; for example, the 20-mer sequence yielded 15 hexamers. The frequency of each k-mer was determined and normalized by the sum of all frequencies. With $k$ denoting each k-mer, the following formula defines relative frequency: relative frequency $(k)$ $=$ normalized frequency $(k)_{\text {Biccl }} /$ normalized frequency $(k)$ Control. To identify Bicc1bound sequence elements, we collected the hexamers whose $Z$-score for the relative frequency was $>3.0$. The enriched hexamers were aligned by Clustal Omega ${ }^{46}$ with default parameters. The motif logo was generated from the aligned hexamers by Weblogo $3^{47}$.

Metagene analysis. Mouse $3^{\prime}$-UTR sequences $(\mathrm{mm} 10)$ were obtained from the UCSC Table Browser by specifying the track as ALL GENCODE V22 and the table as Basic. The 200-nt proximal regions were extracted, and duplicated sequences were removed. The number of motifs present in each region was then counted.

Multiple alignment. Sequences were aligned by MAFFT ${ }^{48}$ or MAFFT at the MPI Bioinformatics Toolkit ${ }^{49}$ with manual changes to align positions of GAC motifs among species. Multiple alignment and folding of RNA were performed by LocARNA ${ }^{50}$.

In vitro transcription. The plasmid pEFSA Dand5-3'UTR was used as a PCR template to generate SP6 transcription matrices. Briefly, a forward primer containing the SP6 promoter sequence and a Reverse primer were used for the amplification of the DNA fragment encoding the sequence of interest using the Phire Green Hot Start II PCR Master Mix (ThermoFisher F126L). For future annealing of a fluorescent probe, the complementary $5^{\prime}$-TGTCTGGGCAACAGGCTCAGG- $3^{\prime}$ sequence was introduced as a $3^{\prime}$ tag via the Reverse primer. For the short transcripts Dand5 $3^{\prime} U T R_{66-110}$ and Dand5 $3^{\prime} U T R_{226-270}$, the transcription matrices were prepared without plasmid template using overlapping PCR primers carrying the mutations of interest. After agarose gel electrophoresis, PCR amplicons were purified on NucleoSpin ${ }^{\oplus}$ Gel and PCR Clean-up (Macherey-Nagel, Cat. No. 740609). 500 ng of SP6 transcription matrice was used to in vitro transcribe the RNA of interest by using the SP6 RNA polymerase kit according to the manufacturer's conditions (Roche, Cat.No. 10810274 001). After 2 hours of incubation at $37^{\circ} \mathrm{C}$, the transcripts were incubated for one additional hour in presence of DNAse I (Roche, Cat.No. 04716728 001) in order to eliminate the SP6 DNA matrix. Finally, the transcripts were purified on Quick Spin Columns (Roche, Cat. No. 11274015 001).

Fluorescent EMSA. The fluorescent DNA probe 5'-CTGAGCCTGTTGCCCAGAC- $3^{\prime}$ carrying a $5^{\prime}$-Dynomics 681 dye, was synthesized by Microsynth AG. Before each experiment, $3^{\prime}$ tagged RNA and the fluorescent DNA probe were preannealed by denaturation $\left(3 \mathrm{~min}\right.$ at $98^{\circ} \mathrm{C}$ ) and renaturation for $10 \mathrm{~min}$ at room temperature. One pmol of $3^{\prime}$-tagged RNA and $2.5 \mathrm{pmol}$ of fluorescent probe were mixed for each condition. Complexes of fluorescent RNA:DNA duplex with recombinant GST-KH were assembled in a final volume of $20 \mu \mathrm{L}$ containing $10 \mathrm{mM}$ Tris- $\mathrm{HCl} \mathrm{pH8,} 100 \mathrm{mM} \mathrm{KCl}, 2.5 \mathrm{mM} \mathrm{MgCl} 2,2.5 \%$ glycerol, $1 \mathrm{mM}$ DTT, and $1 \mu \mathrm{g}$ of yeast tRNAs by incubation on ice for $30 \mathrm{~min}$ in the dark. For competition experiments, the recombinant GST-KH protein was used at a fixed concentration $(200 \mathrm{nM})$. Increasing amounts of unlabeled competitor RNAs were added to pre-assembled fluorescent complexes and incubated for another $30 \mathrm{~min}$. The complexes were then resolved by electrophoresis on a $5 \%$ native polyacrylamide gel containing $45 \mathrm{mM}$ Tris-Borate, $1 \mathrm{mM}$ EDTA, and 2.5\% glycerol. The fluorescence was detected using the Odyssey CLx Infrared Imaging System (LI-COR Biosciences).

Reporting summary. Further information on research design is available in the Nature Research Reporting Summary linked to this article.

\section{Data availability}

Sequencing data of this study were deposited to the Gene Expression Omnibus (GEO) database under accession number GSE140931. Publicly available data was downloaded from NCBI Data Base (https://www.ncbi.nlm.nih.gov), UCSC Table Browser (https:// genome.ucsc.edu). Source data are provided with this paper.

\section{Code availability}

Raw data for biochemical experiments are provided in Source data. The custom script of $\mathrm{K}$-mer analysis is avaliable in the Github page (https://github.com/KRK13/Kmer2021) Other data and codes are available from the corresponding authors upon reasonable request.

Received: 14 January 2020; Accepted: 9 June 2021;

Published online: 01 July 2021

\section{References}

1. Blum, M., Feistel, K., Thumberger, T. \& Schweickert, A. The evolution and conservation of left-right patterning mechanisms. Development 141, 1603-1613 (2014)

2. Nakamura, T. \& Hamada, H. Left-right patterning: conserved and divergent mechanisms. Development 139, 3257-3262 (2012).

3. Pennekamp, P. et al. The ion channel polycystin-2 is required for left-right axis determination in mice. Curr. Biol. 12, 938-943 (2002).

4. Field, S. et al. Pkd1ll establishes left-right asymmetry and physically interacts with Pkd2. Development 138, 1131-1142 (2011).

5. Yoshiba, S. et al. Cilia at the node of mouse embryos sense fluid flow for leftright determination via Pkd2. Science 338, 226-231 (2012).

6. Mizuno, K. et al. Role of $\mathrm{Ca}(2+)$ transients at the node of the mouse embryo in breaking of left-right symmetry. Sci. Adv. 6, eaba1195 (2020).

7. Shiratori, H. \& Hamada, H. TGFbeta signaling in establishing left-right asymmetry. Semin Cell Dev. Biol. 32, 80-84 (2014).

8. Marques, S. et al. The activity of the Nodal antagonist Cerl-2 in the mouse node is required for correct L/R body axis. Genes Dev. 18, 2342-2347 (2004).

9. Kawasumi, A. et al. Left-right asymmetry in the level of active Nodal protein produced in the node is translated into left-right asymmetry in the lateral plate of mouse embryos. Dev. Biol. 353, 321-330 (2011).

10. Maisonneuve, C. et al. Bicaudal C, a novel regulator of Dvl signaling abutting RNA-processing bodies, controls cilia orientation and leftward flow. Development 136, 3019-3030 (2009).

11. Piazzon, N., Maisonneuve, C., Guilleret, I., Rotman, S. \& Constam, D. B. Biccl links the regulation of cAMP signaling in polycystic kidneys to microRNAinduced gene silencing. J. Mol. Cell Biol. 4, 398-408 (2012).

12. Rothe, B. et al. Biccl Polymerization Regulates the Localization and Silencing of Bound mRNA. Mol. Cell Biol. 35, 3339-3353 (2015).

13. Doidge, R., Mittal, S., Aslam, A. \& Winkler, G. S. Deadenylation of cytoplasmic mRNA by the mammalian Ccr4-Not complex. Biochem Soc. Trans. 40, 896-901 (2012).

14. Collart, M. A. \& Panasenko, O. O. The Ccr4-not complex. Gene 492, 42-53 (2012).

15. Chicoine, J. et al. Bicaudal-C recruits CCR4-NOT deadenylase to target mRNAs and regulates oogenesis, cytoskeletal organization, and its own expression. Dev. Cell 13, 691-704 (2007).

16. Suzuki, T. et al. Postnatal liver functional maturation requires Cnot complexmediated decay of mRNAs encoding cell cycle and immature liver genes. Development 146, dev168146(2019).

17. Morita, M. et al. Depletion of mammalian CCR4b deadenylase triggers elevation of the p27Kip1 mRNA level and impairs cell growth. Mol. Cell Biol. 27, 4980-4990 (2007).

18. Shinohara, K. et al. Two rotating cilia in the node cavity are sufficient to break left-right symmetry in the mouse embryo. Nat. Commun. 3, 622 (2012).

19. Supp, D. M., Witte, D. P., Potter, S. S. \& Brueckner, M. Mutation of an axonemal dynein affects left-right asymmetry in inversus viscerum mice. Nature 389, 963-966 (1997).

20. Nonaka, S., Shiratori, H., Saijoh, Y. \& Hamada, H. Determination of left-right patterning of the mouse embryo by artificial nodal flow. Nature 418, 96-99 (2002).

21. Maerker, M. et al. Biccl and Dicer regulate left-right patterning through posttranscriptional control of the Nodal-inhibitor Dand5. Nat. Commun. In Press.

22. Thastrup, O., Cullen, P. J., Drobak, B. K., Hanley, M. R. \& Dawson, A. P. Thapsigargin, a tumor promoter, discharges intracellular $\mathrm{Ca} 2+$ stores by specific inhibition of the endoplasmic reticulum Ca2(+)-ATPase. Proc. Natl Acad. Sci. USA 87, 2466-2470 (1990).

23. Nicastro, G., Taylor, I. A. \& Ramos, A. KH-RNA interactions: back in the groove. Curr. Opin. Struct. Biol. 30, 63-70 (2015).

24. Nakamura, T. et al. Fluid flow and interlinked feedback loops establish leftright asymmetric decay of Cerl2 mRNA. Nat. Commun. 3, 1322 (2012).

25. Hollingworth, D. et al. KH domains with impaired nucleic acid binding as a tool for functional analysis. Nucleic Acids Res 40, 6873-6886 (2012).

26. Lambert, $N$. et al. RNA Bind-n-Seq: quantitative assessment of the sequence and structural binding specificity of RNA binding proteins. Mol. Cell 54, 887-900 (2014).

27. Zhang, Y. et al. Bicaudal-C spatially controls translation of vertebrate maternal mRNAs. RNA 19, 1575-1582 (2013).

28. Leal-Esteban, L. C., Rothe, B., Fortier, S., Isenschmid, M. \& Constam, D. B. Role of Bicaudal C1 in renal gluconeogenesis and its novel interaction with the CTLH complex. PLoS Genet 14, e1007487 (2018).

29. Li, X. et al. Adipocyte-specific disruption of mouse Cnot 3 causes lipodystrophy. FEBS Lett. 591, 358-368 (2017).

30. Ukita, K. et al. Wnt signaling maintains the notochord fate for progenitor cells and supports the posterior extension of the notochord. Mech. Dev. 126, 791-803 (2009). 
31. Nakamura, T. et al. Generation of robust left-right asymmetry in the mouse embryo requires a self-enhancement and lateral-inhibition system. Dev. Cell 11, 495-504 (2006).

32. Dowdle, M. E. et al. A single KH domain in Bicaudal-C links mRNA binding and translational repression functions to maternal development. Development 146, dev172486 (2019)

33. Nakel, K., Hartung, S. A., Bonneau, F., Eckmann, C. R. \& Conti, E. Four KH domains of the C. elegans Bicaudal-C ortholog GLD-3 form a globular structural platform. RNA 16, 2058-2067 (2010).

34. Beuth, B., Pennell, S., Arnvig, K. B., Martin, S. R. \& Taylor, I. A. Structure of a Mycobacterium tuberculosis NusA-RNA complex. EMBO J. 24, 3576-3587 (2005).

35. Park, S., Blaser, S., Marchal, M. A., Houston, D. W. \& Sheets, M. D. A gradient of maternal Bicaudal-C controls vertebrate embryogenesis via translational repression of mRNAs encoding cell fate regulators. Development 143, 864-871 (2016).

36. Tran, U. et al. The RNA-binding protein bicaudal $C$ regulates polycystin 2 in the kidney by antagonizing miR-17 activity. Development 137, 1107-1116 (2010).

37. Iwakawa, H. O. \& Tomari, Y. The Functions of MicroRNAs: mRNA Decay and Translational Repression. Trends Cell Biol. 25, 651-665 (2015).

38. Behm-Ansmant, I. et al. mRNA degradation by miRNAs and GW182 requires both CCR4:NOT deadenylase and DCP1:DCP2 decapping complexes. Genes Dev. 20, 1885-1898 (2006)

39. Jonas, S. \& Izaurralde, E. Towards a molecular understanding of microRNAmediated gene silencing. Nat. Rev. Genet 16, 421-433 (2015).

40. Takao, D. et al. Asymmetric distribution of dynamic calcium signals in the node of mouse embryo during left-right axis formation. Dev. Biol. 376, 23-30 (2013).

41. Mochizuki, T. et al. Cloning of inv, a gene that controls left/right asymmetry and kidney development. Nature 395, 177-181 (1998).

42. Watanabe, D. et al. The left-right determinant Inversin is a component of node monocilia and other $9+0$ cilia. Development 130, 1725-1734 (2003).

43. Wilkinson, D. G. \& Nieto, M. A. Detection of messenger RNA by in situ hybridization to tissue sections and whole mounts. Methods Enzymol. 225, 361-373 (1993).

44. Minegishi, K. et al. A Wnt5 activity asymmetry and intercellular signaling via PCP proteins polarize node cells for left-right symmetry breaking. Dev. Cell 40, 439-452 (2017).

45. Hashimoto, M. et al. Planar polarization of node cells determines the rotational axis of node cilia. Nat. Cell Biol. 12, 170-176 (2010).

46. Sievers, F. et al. Fast, scalable generation of high-quality protein multiple sequence alignment using Clustral Omega. Mol. Syst. Biol. 7, 539 (2011).

47. Crooks, G. E., Hon, G., Chandonia, J.-M. \& Brenner, S. E. WebLogo: a sequence logo generator. Genome Res 14, 1188-1190 (2004).

48. Katoh, K. \& Standley, D. M. MAFFT multiple sequence alignment software version 7: improvements in performance and usability. Mol. Biol. Evol. 30, 772-780 (2013).

49. Zimmermann, L. et al. A completely reimplemented MPI bioinformatics toolkit with a new HHpred server at its core. J. Mol. Biol. 430, 2237-2243 (2018).

50. Will, S., Reiche, K., Hofacker, I. L., Stadler, P. F. \& Backofen, R. Inferring noncoding RNA families and classes by means of genome-scale structurebased clustering. PLoS Comput. Biol. 3, e65 (2007).

\section{Acknowledgements}

We thank K. Okamoto (University of Tokyo) for PIV analysis software; Y. Igarashi for the software for quantitative analysis of basal body position; H. Sasaki for Noto CreERT2/+ mice; and M. Yamagata and S. Wada for technical support and designing oligonucleo- tides for RBNS analysis, respectively. This study was supported by grants from the Ministry of Education, Culture, Sports, Science, and Technology (MEXT) of Japan (no. 17H01435), from Core Research for Evolutional Science and Technology (CREST) of the Japan Science and Technology Agency (no. JPMJCR13W5) and from the Takeda Science Foundation to H.H.; by a Grant-in-Aid (no. 18K14725) for Early-Career Scientists from the Japan Society for the Promotion of Science (JSPS), a Kakehashi grant from BDROtsuka Pharmaceutical Collaboration Center, and a research grant (no. 2018M-018) from the Kato Memorial Bioscience Foundation to K.M.; by grants from the NIBB Individual Collaborative Research Program (nos. 16-312 and 17-316) and a Sinergia grant (no. CRSI33_130662) from the Swiss National Science Foundation to D.B.C.; and by a KAKENHI grant (no. 15H05722) from JSPS and a research grant from The Mitsubishi Foundation to H.S.

\section{Author contributions}

K.M. performed most experiments with mouse embryos. Y.I., H.N., and K.T. generated transgenic and mutant mice. B.R. performed biochemical analysis of Biccl and Cnot3. K.R.K., E.M., and H.O. performed RBNS analysis. K.B. and H.K. performed transgenic assays with deletion mutants. T.Y. examined the phenotype of Cnot $3 \mathrm{CKO}$ mutant mice T.A. K. and E.K. performed experiments with Pkd2 mutant embryos and Dand5 $\Delta 200$ mutant embryos, respectively. X.S. examined the localization of Cnot3 protein in mouse embryos. H.H., D.B.C., H.S., K.M., B.R., and K.R.K. conceived the project and wrote the paper. All authors contributed to the revision of the manuscript.

\section{Competing interests}

The authors declare no competing interests.

\section{Additional information}

Supplementary information The online version contains supplementary material available at https://doi.org/10.1038/s41467-021-24295-2.

Correspondence and requests for materials should be addressed to H.S., D.B.C. or H.H

Peer review information Nature Communications thanks Matthew Stower, Oliver Wessely and the other, anonymous, reviewer(s) for their contribution to the peer review of this work.

Reprints and permission information is available at http://www.nature.com/reprints

Publisher's note Springer Nature remains neutral with regard to jurisdictional claims in published maps and institutional affiliations.

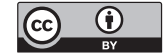

Open Access This article is licensed under a Creative Commons Attribution 4.0 International License, which permits use, sharing, adaptation, distribution and reproduction in any medium or format, as long as you give appropriate credit to the original author(s) and the source, provide a link to the Creative Commons license, and indicate if changes were made. The images or other third party material in this article are included in the article's Creative Commons license, unless indicated otherwise in a credit line to the material. If material is not included in the article's Creative Commons license and your intended use is not permitted by statutory regulation or exceeds the permitted use, you will need to obtain permission directly from the copyright holder. To view a copy of this license, visit http://creativecommons.org/ licenses/by/4.0/.

(c) The Author(s) 2021 